ARTíCUlo

\title{
Efecto del tamaño de tamiz en la caracterización de la macrofauna marina bentónica: Implicancias para su uso en líneas de base, caracterizaciones preliminares de sitios para la acuicultura y monitoreos ambientales en Chile
}

Effects of sieve mesh size on the characterization of marine benthic macrofauna: Implications for their use in baselines, preliminary characterization of aquaculture sites and environmental monitoring in Chile

\section{Eduardo Hernández-Miranda ${ }^{\circledR 1,2,3^{*}}$, Rodrigo Veas ${ }^{\circledR 2,3}$, M. Cristina Krautz ${ }^{\circledR 2}$, Nelson Hidalgo ${ }^{2}$, Filoromo San Martín ${ }^{2,3}$ y Renato A. Quiñones ${ }^{\circledR 1,3,4}$}

${ }^{1}$ Centro Interdisciplinario para la Investigación Acuícola (INCAR), Universidad de Concepción, Casilla 160-C, Concepción, Chile ${ }^{2}$ Laboratorio de Investigación en Ecosistemas Acuáticos (LInEA), Facultad de Ciencias Naturales \& Oceanográficas, Universidad de Concepción, Casilla 160-C, Concepción, Chile

${ }^{3}$ Programa de Estudios Ecosistémicos del Golfo de Arauco (PREGA), Facultad de Ciencias Naturales \& Oceanográficas, Universidad de Concepción, Casilla 160-C, Concepción, Chile

${ }^{4}$ Departamento de Oceanografía, Facultad de Ciencias Naturales y Oceanográficas, Universidad de Concepción, Casilla 160-C, Concepción, Chile

*Autor corresponsal: eduhernandez@udec.cl

Abstract.- The ecological characteristics of the benthic macrofauna make a good indicator to evaluate the effects of anthropic perturbations (e.g., aquaculture, submarine pipelines emissions) on marine ecosystems. Chilean environmental regulation establishes that macrofauna characterization must be performed with sieves with 1,000 $\mu \mathrm{m}$ opening. To evaluate the loss of information on species composition and abundance by using an opening of 1,000 $\mu \mathrm{m}$ instead of $500 \mu \mathrm{m}$, subtidal samplings were performed during summer and winter of 2018 in 5 areas of the Gulf of Arauco, central Chile. Sampling and analysis consisted of: (i) samples were sieved through 1,000 $\mu \mathrm{m}$ mesh and the organisms passing through this sieve were retained in $500 \mu \mathrm{m}$ mesh sieves; (ii) identifying the taxa retained in both sieves to the lowest possible level, and (iii) comparison of the macrofauna between sieves, periods and sampling areas, using univariate and multivariate statistics. The analyses showed significant differences in the community structure among sieve sizes, areas and sampling periods. The AMBI and W indexes, used as community bio-indicators, also showed significant differences between sieve sizes. The results revealed that, although a 1,000 $\mu \mathrm{m}$ sieve can identify spatial and temporal differences in the macrofauna, it underestimates the level of impact shown by community bio-indicators. The use of a $500 \mu \mathrm{m}$ sieve is recommended if the objective is to evaluate anthropic impact on the marine environment, especially for baseline studies, preliminary site characterizations for aquaculture, monitoring and/or environmental surveillance programmes. This information should also be complemented with multivariate statistical tools that are capable of incorporating together all the biological and environmental information compiled in the study area.

Key words: Ecological status, communities, biodiversity, indicators, environmental impact evaluation system, conservation

Resumen.- Las características ecológicas de la macrofauna bentónica, la convierten en un buen indicador para evaluar los efectos de perturbaciones antropogénicas (e.g., acuicultura, emisarios submarinos, etc.) sobre los ecosistemas marinos. La normativa chilena aplicable a monitoreos ambientales establece que su caracterización debe realizarse utilizando tamices de $1.000 \mu \mathrm{m}$ de abertura. Con el objetivo de evaluar la pérdida de información en composición y abundancia de las especies al utilizar este tamiz respecto de uno de $500 \mu \mathrm{m}$, se realizaron muestreos submareales en invierno y verano del 2018 en 5 áreas del Golfo de Arauco, Chile central. El muestreo y análisis consistió en: (i) cernido en tamiz de $1.000 \mu \mathrm{m}$, reteniendo los organismos que traspasan este tamiz en uno de 500 $\mu \mathrm{m}$, (ii) identificación de los organismos retenidos en ambos tamices hasta el nivel taxonómico más bajo posible y, (iii) comparación de la macrofauna entre tamices, períodos y áreas de muestreo, utilizando estadística univariada y multivariada. A partir de los análisis se encontraron diferencias significativas en la estructura comunitaria de la macrofauna entre tamices, áreas y periodos de muestreo. Los índices AMBI y W, utilizados como bio-indicadores comunitarios, también identifican diferencias significativas entre tamices. Los resultados señalan que, si bien el uso de un tamiz de $1.000 \mu \mathrm{m}$ permite identificar diferencias espaciales y temporales en la macrofauna, con su uso se subestima el nivel de impacto detectado por los bio-indicadores comunitarios. Por tal razón, se recomienda utilizar un tamiz de $500 \mu \mathrm{m}$ si el objetivo es evaluar impactos antropogénicos en el ambiente marino, especialmente en líneas de base, caracterizaciones preliminares de sitio para la acuicultura y programas de vigilancia ambiental. Toda la información, debiera, además, ser integrada mediante herramientas estadísticas multivariadas, capaces de incorporar en conjunto los antecedentes biológicos y ambientales recopilados en el área de estudio. 


\section{INTRODUCCIÓN}

La optimización de metodologías de muestreo, obtención y análisis de información y, las herramientas estadísticas utilizadas en estudios que tienen como objetivo evaluar el impacto ambiental producto de perturbaciones antropogénicas en ecosistemas marinos, son parte de un proceso continuo y de alta relevancia societal. Desde el punto de vista ecológico, se reconoce que una correcta evaluación de un impacto antropogénico en el ambiente, depende de una buena caracterización previa (i.e., Línea de Base) de la biodiversidad del área afectada (Dauvin et al. 2010). Una correcta identificación taxonómica, permite que la caracterización de patrones espaciales y de la variabilidad temporal natural de las abundancias poblacionales de las especies residentes en un área particular (Kingsford et al. 1998, Scheiner \& Willig 2008), constituyan una robusta fuente de información para generar hipótesis, diseñar experimentos específicos, gestionar decisiones ambientales y establecer correctos programas de monitoreo y/o investigaciones específicas a lo largo del tiempo (Green 1979, Kennelly \& Underwood 1984, Andrew \& Mapstone 1987, James et al. 1995, Kennedy \& Cheong 2013).

Entre los modelos biológicos más utilizados en evaluaciones de impacto ambiental en ambientes marinos, se encuentra la macrofauna bentónica (Bilyard 1987, Harris 2012). Los resultados obtenidos a partir de su caracterización son dependientes de la medida de abertura de la malla o tamiz utilizado para la retención de los organismos (Schlacher \& Wooldridge 1996, Barba et al. 2010, Couto et al. 2010, Solana-Arellano et al. 2014, Rubio-Polania et al. 2016). La elección del tamaño de abertura es un tema debatible, ya que, si bien un tamiz grueso ayuda a retener la fracción de mayor tamaño del material recolectado, subestima el número y presencia de organismos más pequeños. Por el contrario, tamices de menor abertura pueden retener mayor cantidad de organismos, pero los costos de procesamiento debido al mayor tiempo invertido en la revisión, son más elevados (de Souza \& Barros 2017). En la actualidad, se discute sobre el compromiso tiempo/costo de diferentes opciones para el análisis de este tipo de muestras biológicas y, de la calidad/cantidad de información necesaria para evaluar adecuadamente el impacto ambiental que se haya generado o podría generarse a causa de alguna actividad antropogénica en los ecosistemas marinos (Carey \& Keough 2002, Souza \& Barros 2015, de Souza \& Barros 2017). No obstante el uso generalizado de tamices en estudios que caracterizan la macrofauna marina bentónica, existen pocos trabajos donde se compare cualitativa y cuantitativamente los resultados obtenidos de su estructura comunitaria, considerando las ventajas y desventajas del tamaño disímil de los individuos retenidos por tamices de diferente abertura (Bachelet 1990, Edgar 1990, Schlacher \& Wooldridge 1996, Ramsay et al.
1997, Hudson \& Adams 1998, Tanaka \& Leite 1998, Morin et al. 2004, Lampadariou et al. 2005, Souza \& Barros 2015, Pino et al. 2015, de Souza \& Barros 2017).

El tamaño de abertura del tamiz en estudios de macrofauna marina bentónica es generalmente establecido entre $0,5 \mathrm{~mm}(=500 \mu \mathrm{m})$ y $1 \mathrm{~mm}(=1.000 \mu \mathrm{m})$ (Eleftheriou \& Holme 1984, Kingston \& Riddle 1989, James et al. 1995). Su elección dependerá de los objetivos del estudio, del tipo de sustrato y de los organismos de interés, por ejemplo, se recomienda utilizar tamices de menor abertura si se requiere retener mayoritariamente estadíos juveniles de la macrofauna, principalmente poliquetos (Reish 1959, Eleftheriou \& Holme 1984, Bishop \& Hartley 1986, Bachelet 1990, James et al. 1995). En este contexto, si el objetivo del estudio está orientado a caracterizar los impactos de alguna intervención antropogénica, el tamaño de abertura del tamiz debiera ser seleccionado de acuerdo a los requerimientos de los indicadores a utilizar. Desde el punto de vista ambiental es posible reconocer indicadores biológicos (bio-indicadores) y ecológicos (Word 1979, Dauvin \& Ruellet 2007, Dauvin et al. 2010, Beauchard et al. 2017). Los primeros, se focalizan en la evaluación de respuestas específicas de los organismos ante una perturbación, en niveles que van desde lo celular, hasta lo comunitario; mientras que los segundos, se orientan además a evaluar los patrones de respuesta emergentes, que se observan en el ecosistema o en parte de sus componentes, relacionados a los cambios naturales y/o antropogénicos de las condiciones ambientales (Dauvin et al. 2010).

Los bio-indicadores comunitarios (AZTI Marine Biotic Index (AMBI), ITI, BOPA, entre otros; Word 1979, Borja et al. 2000, Dauvin \& Ruellet 2007) son obtenidos a partir de la presencia y abundancia de los organismos en un hábitat específico, dando cuenta del grado de perturbación, contaminación o del estatus ecológico de ese ambiente, asignando las especies a categorías de acuerdo a niveles de sensibilidad (Grall \& Glémarec 1997), grupos tróficos (Word 1979) o razones de sus abundancias (Dauvin \& Ruellet 2007). Un bio-indicador comunitario ideal es aquel que exprese una estrecha relación entre la tolerancia ambiental de los organismos y la fuente de perturbación, es decir, que presente una alta sensibilidad a la alteración de los factores físicos y químicos del medio que habitan (Morales 2011, Beauchard et al. 2017). Entre los bio-indicadores comunitarios más utilizados en ambientes marinos destacan aquellos obtenidos a partir de la caracterización de la macrofauna bentónica. Estos organismos presentan ventajosas cualidades para evaluar la calidad ambiental, entre ellas, ser sésiles o de reducida movilidad, contener especies con respuestas disímiles a la contaminación y presentar ciclos de vida que van desde meses hasta años, lo cual permite integrar efectos negativos en el ambiente por 
largos períodos de tiempo y en lugares específicos (Pearson \& Rosenberg 1978, Gray et al. 1988, Carrasco \& Gallardo 1989, Levin et al. 2001, Paul et al. 2001, Dauvin 2007).

Los bio-indicadores comunitarios debieran, además, complementarse con la información ecológica obtenida de la distribución y abundancia de las especies presentes en el área de estudio, utilizando antecedentes univariados (e.g., riqueza de especies/taxa, diversidad alfa) y multivariados (e.g., diversidad beta, disimilitud multivariada) (Clarke \& Warwick 2001, Anderson et al. 2006, 2011). En conjunto, toda la información debiese permitir discriminar los efectos de una perturbación de origen antropogénico, respecto de otras de tipo natural (e.g., tsunamis, hipoxias naturales, erupciones volcánicas, etc.), entregando elementos de juicio objetivos para evaluar posibles riesgos ambientales para los otros componentes del ecosistema y los seres humanos. Así, el monitoreo de los cambios en la estructura comunitaria de la macrofauna bentónica y sus bio-indicadores se convierten en una herramienta robusta para la evaluación de efectos antropogénicos sobre los ecosistemas marinos (Word 1979, Borja et al. 2000, Clarke \& Warwick 2001, Dauvin \& Ruellet 2007, Marques et al. 2013).

En Chile, de acuerdo a la Resolución Exenta $\mathrm{N}^{\circ} 3612 / 2009^{1}$, el análisis de la macrofauna marina bentónica, con la finalidad de realizar una Caracterización Preliminar de Sitios (CPS) y la subsecuente Información Ambiental (INFA) en áreas destinadas a la acuicultura, considera el uso de un tamiz de $1 \mathrm{~mm}(1.000 \mu \mathrm{m})$ de abertura para la separación, y posterior identificación y cuantificación de los organismos. Esta Resolución es utilizada, además, como metodología estándar por laboratorios autorizados como Entidades Técnicas de Fiscalización Ambiental (ETFA) (e.g., acreditados por la NCh $17.020^{2}$ y/o NCh. $\left.17.025^{3}\right)$, para los alcances relacionados con el monitoreo de la macrofauna marina bentónica en todo tipo de proyectos que requieran reportar información proveniente de sus Programas de Vigilancia Ambiental (PVA), exigidos por una Resolución de Calificación Ambiental (RCA) o, a solicitud de la Superintendencia del Medio Ambiente, todo esto en el marco del Sistema de Evaluación de Impacto Ambiental vigente (Ley $19.300^{4}$ y Ley $20.417^{5}$ ). La metodología descrita en esta Resolución, sin embargo, no tiene vinculación específica respecto a los bio-indicadores comunitarios que se pudieran utilizar posteriormente en la evaluación de los impactos de un proceso productivo particular. En específico sólo hace referencia a un grupo de organismos sobre los cuales se debiera alcanzar un mayor nivel de resolución taxonómica en la identificación (i.e., a nivel de género), entre ellas, los poliquetos de las familias Capitellidae, Oweniidae y Cirratulidae, además de los gastrópodos de la familia Nassaridae.

Con el objetivo de evaluar la pérdida de información en composición, abundancia y biomasa de las especies de la macrofauna al utilizar un tamiz de $1.000 \mu \mathrm{m}$ respecto de uno de $500 \mu \mathrm{m}$, y su efecto en la estimación de dos bio-indicadores comunitarios, se realizaron muestreos submareales bentónicos en el Golfo de Arauco. En específico el estudio se orientó a: (i) Determinar si el tamaño de abertura del tamiz $(500 \mu \mathrm{m}$ y $1.000 \mu \mathrm{m})$ por separado permite identificar diferencias espaciales y temporales en parámetros ecológicos de la macrofauna, (ii) Evaluar la presencia de diferencias espaciales y temporales en las abundancias y biomasas de la macrofauna entre ambos tamices y (iii) Establecer relaciones entre los patrones univariados y multivariados encontrados para cada tamiz y los bio-indicadores comunitarios seleccionados.

\footnotetext{
${ }^{1}$ Res. Ex. 3612/2009. Resolución que fija las metodologías para elaborar la caracterización preliminar de sitio (CPS) y la información ambiental (INFA) Ministerio de Economía, Fomento y Reconstrucción; Subsecretaría de Pesca (modificada 06.04.2020 Res. Ex. 905), Santiago de Chile.

${ }^{2}$ NCh-ISO 17020/2012. Evaluación de la conformidad - requisitos para el funcionamiento de diferentes tipos de organismos que realizan la inspección. Instituto Nacional de Normalización, Santiago de Chile.

${ }^{3} \mathrm{NCh}$-ISO 17025/2005. Requisitos generales para la competencia de los laboratorios de ensayo y calibración. Instituto Nacional de Normalización, Santiago de Chile.

${ }^{4}$ Ley N ${ }^{\circ}$ 19.300. Ley Sobre Bases Generales del Medio Ambiente. Ministerio Secretaría General de la Presidencia, Santiago de Chile.

${ }^{5}$ Ley N²0.417. Crea el Ministerio, el Servicio de Evaluación Ambiental y la Superintendencia del Medio Ambiente. Ministerio Secretaría General de la Presidencia, Santiago de Chile.
} 


\section{MATERIALES Y MÉTODOS}

\section{TOMA DE MUESTRAS, PROCESAMIENTO Y ANÁLISIS EN LABORATORIO}

Durante el invierno y verano del 2018, en 5 áreas submareales del sector costero oriental del Golfo de Arauco, Chile central, se obtuvieron muestras de sedimento marino para la identificación y cuantificación de la macrofauna bentónica. Las muestras fueron recolectadas mediante un toma testigo HAPS Corer de $0,01 \mathrm{~m}^{2}$ de área. La selección de las áreas para este estudio consideró cubrir un gradiente ambiental de los sedimentos en una zona de similar batimetría (10 a $15 \mathrm{~m})$. Algunas características físico-químicas de los sedimentos y del estrato profundo de la columna de agua para las 5 áreas de estudio se presentan en la Tabla 1. En cada área de muestreo se recolectaron 4 réplicas, que se tamizaron a bordo de la embarcación con agua de mar utilizando un tamiz de $500 \mu \mathrm{m}$ de abertura de malla. Las muestras fueron preservadas con formalina tamponada al $10 \%$ con borato de sodio ( 3 muestras) y etanol al 90\% (una muestra). En laboratorio las muestras fueron nuevamente lavadas y procesadas utilizando 2 tamices sobrepuestos: el primero con una abertura de $1.000 \mu \mathrm{m}$ y el segundo con una de $500 \mu \mathrm{m}$ (recepción final de la muestra). El contenido retenido en ambos tamices fue separado en frascos independientes y preservados en alcohol al 90\%, señalando rótulos de área de muestreo, número de réplica, fecha, fracción del tamiz utilizado y preservante original. Mediante una lupa estereoscópica, se identificaron y cuantificaron los organismos hasta el nivel taxonómico más bajo posible (definidos en adelante como taxa o taxon), determinando, además, el peso húmedo de cada uno de ellos por réplica, mediante una balanza analítica de $0,0001 \mathrm{~g}$ de sensibilidad. Con esta información, se generó una base de datos para la abundancia (ind. 0,01 $\mathrm{m}^{-2}$ ) y otra para la biomasa $\left(\mathrm{g} 0,01 \mathrm{~m}^{-2}\right)$ de cada taxon. $\mathrm{La}$ base de datos comparativa para los análisis posteriores entre tamices consistió en (i) la suma de organismos retenidos en los tamices de 500 y $1.000 \mu \mathrm{m}$ (en adelante, tamiz de $500 \mu \mathrm{m}$ ) y (ii) organismos retenidos en tamiz de $1.000 \mu \mathrm{m}$ (en adelante, tamiz de $1.000 \mu \mathrm{m}$ ).

\section{ANÁLISIS ESTADÍSTICOS: DIVERSIDAD UNIVARIADA, MULTIVARIADA Y BIO-INDICADORES}

Con el objetivo de evaluar diferencias en la macrofauna bentónica entre tamices $(500 \mu \mathrm{m}$ y $1.000 \mu \mathrm{m})$, período (invierno y verano), y área de muestreo (5 áreas) se utilizaron las siguientes variables comunitarias univariadas: abundancia y biomasa total, riqueza de especies/taxa (S), diversidad $\log _{\mathrm{e}}$ Shannon-Wiener (H'),
Tabla 1. Valores promedio ( \pm desviación estándar) de variables ambientales medidas en la columna de agua cerca del fondo y los sedimentos marinos en 5 áreas de muestreo en el Golfo de Arauco durante el invierno y verano del 2018 / Mean values ( \pm standard deviation) of environmental variables measured in the water column close to the sea bottom and in the marine sediments from 5 sampling areas in the Gulf of Arauco registered in winter and summer of 2018

\begin{tabular}{lcc}
\hline & Invierno & Verano \\
\hline Columna de agua & & \\
Temperatura $\left({ }^{\circ} \mathrm{C}\right)$ & $12,4 \pm 0,0$ & $13,4 \pm 0,2$ \\
Salinidad & $34,2 \pm 0,0$ & $34,2 \pm 0,1$ \\
Oxígeno disuelto $\left(\mathrm{mL} \mathrm{L}^{-1}\right)$ & $2,7 \pm 0,2$ & $4,0 \pm 0,2$ \\
Sedimentos & & \\
Media $(\phi)$ & 2,104 & 2,646 \\
Sorting $(\phi)$ & 1,276 & 1,636 \\
Grava $(\%)$ & $4,96 \%$ & $2,34 \%$ \\
Arena $(\%)$ & $93,54 \%$ & $90,36 \%$ \\
Limo-Arcilla $(\%)$ & $1,52 \%$ & $7,28 \%$ \\
MOT $(\%)$ & $7,12 \pm 2,87$ & $7,03 \pm 3,71$ \\
pH & $7,0 \pm 0,1$ & $7,5 \pm 0,1$ \\
Temperatura $\left({ }^{\circ} \mathrm{C}\right)$ & $12,4 \pm 0,2$ & $12,3 \pm 1,5$ \\
Potencial redox $(\mathrm{mV})$ & $-142,2 \pm 68,8$ & $-282,1 \pm 90,6$ \\
\hline
\end{tabular}

dominancia Simpson (Lambda) y equidad Pielou (J') (sensu Levin \& Gage 1998, Clarke \& Warwick 2001, Magurran 2004); además, se utilizaron las abundancias y biomasas relativas de todas las taxa, para estimar medidas de disimilitud comunitaria multivariada entre las diferentes muestras obtenidas (sensu Anderson et al. 2006, 2011). Gráficamente, las variables univariadas son presentadas como barras con su desviación estándar $\mathrm{y}$, las disimilitudes multivariadas mediante análisis de escalamiento no-métrico multidimensional (nMDS), a partir del índice de similitud de Bray-Curtis con datos transformados a raíz cuarta. Mediante PERMANOVA (Anderson 2001), se evaluaron diferencias significativas en la diversidad univariada para los factores tamiz y período de muestreo. Para la estructura comunitaria multivariada, utilizando similar información que, en los nMDS, análisis PERMANOVA fueron realizados para evaluar (i) si cada tamiz por separado es capaz de determinar diferencias estadísticas espaciales y temporales para la abundancia y biomasa de la macrofauna y, (ii) la presencia de diferencias estadísticas entre tamices, y entre períodos y áreas de muestreo, tanto para la abundancia como para la biomasa de la macrofauna. Las diferencias significativas detectadas por PERMANOVA para cada factor fueron evaluadas a posteriori mediante análisis pareado (pair-wise test), las cuales fueron representadas gráficamente, mediante análisis métrico multidimensional (mMDS), a partir de 
elipses de pseudo-intervalos de confianza al 95\%. Para cada factor de análisis, mediante análisis de ordenación de coordenadas principales (PCO), se representaron las interacciones sitio de muestreo/tamiz y período de muestreo/tamiz. Complementariamente, el análisis de Similitud Porcentual (SIMPER) fue utilizado para determinar las especies que aportaron mayoritariamente a la disimilitud de la macrofauna entre tamices. Los análisis fueron realizados con los programas PRIMER v7 y PERMANOVA+ para PRIMER (Clarke \& Warwick 2001, Clarke \& Gorley 2006, Anderson et al. 2008).

El grado de perturbación del ambiente (i.e., estatus ecológico) se analizó por medio de dos bio-indicadores comunitarios (i) índice AMBI (Borja et al. 2000), software AMBI (AZTI) ${ }^{6}$ v5.0 (Borja et al. 2012), con el listado de especies actualizado hasta mayo 2019 y (ii) índice W (Warwick 1986). Las estimaciones de AMBI siguieron las indicaciones de Borja \& Muxica (2005) y Teixeira et al (2012), con algunas modificaciones, esto dado que algunos taxa identificados en el Golfo de Arauco no se encontraban asignados a los grupos ecológicos (GE) pre-establecidos (Tabla 2). En general, los GE van desde grupo I (especies muy sensibles) hasta grupo V (especies oportunistas de primer orden) (Hily et al. 1986, Grall \& Glémarec 1997, Borja et al. 2000). Las modificaciones, arriba señaladas, consistieron en asignar los taxa inicialmente "no asignados" a algún GE de acuerdo a su relación taxonómica (e.g., familia o género), considerando los siguientes dos criterios (i) el mejor GE (AMBI-1) y, (ii) el peor GE (AMBI-2) al cual pudiera ser asignado cada taxon, obteniéndose así el rango extremo de posibles respuestas. Considerando los lineamientos de AMBI, de los 62 taxa identificados, 6 fueron categorizados como "ignorados" y excluidos de estos análisis. Las abundancias relativas de los 6 taxa "ignorados" corresponden a un 1,26\% (500 $\mu \mathrm{m})$ y a un $1,04 \%(1.000 \mu \mathrm{m})$. De los taxa "no-ignorados" $(\mathrm{N}=$ 56), y a partir de sus abundancias relativas AMBI asignó inicialmente un $78,92 \%$ a un GE en el tamiz de $500 \mu \mathrm{m}$ y un $73,56 \%$ en el de $1.000 \mu \mathrm{m}$ (i.e., taxa presentes en listado de AMBI). Los taxa "no asignados" inicialmente (21,08 y $26,44 \%$, respectivamente), fueron asignados al mejor y peor GE de acuerdo a la familia (17 taxa) o género (7 taxa) al que pertenecen (ver Tabla 2 para detalles). A partir de estos dos criterios el 100\% de los organismos "noignorados" fue asignado a un GE, obteniendo los valores comunitarios para AMBI-1 y 2 como rangos extremos.
Referencialmente, AMBI presenta los siguientes límites de clasificación: entre 0 y 1,2 (No contaminado/Normal), entre 1,2 y 3,3 (Levemente contaminado), entre 3,3 y 5,0 (Medianamente contaminado), entre 5,0 y 6,0 (Fuertemente contaminado) y, desde 6,0 a 7 (Extremadamente contaminado/Azoico) (Borja et al. 2000). El índice W (Clarke 1990, Warwick \& Clarke 1994), por su parte, que es utilizado a partir de las curvas ABC (Abundance Biomass Comparison, Warwick 1986), consiste en un algoritmo que mide la distancia relativa entre las curvas de k-dominancia de abundancia y biomasa, cuya escala va en el rango de $-1 \mathrm{a}+1$. Valores negativos indican que la curva de abundancia se encuentra por sobre la curva de biomasa, mientras que los positivos indican lo contrario. Teóricamente, en ambientes no perturbados, la presencia de organismos de mayor tamaño da como resultado que la curva de biomasa se encuentre por sobre la de abundancia (valores positivos), mientras que, en ambientes altamente perturbados, dominados por organismos de pequeño tamaño (i.e., especies oportunistas de primer orden), la curva de abundancia se sitúa por encima de la curva de biomasa (valores negativos), pudiendo interpretarse numéricamente un gradiente de perturbación entre ambos extremos (Warwick \& Clarke 1994).

Posteriormente, en los análisis PCO se incorporó todas las variables de diversidad univariada, además de las estimaciones de W, AMBI y M-AMBI (Muxika et al. 2007). El análisis M-AMBI integra los indicadores de riqueza de especies/taxa (S) y de diversidad de ShannonWienner (H') junto al de AMBI bajo una perspectiva multivariada. M-AMBI presenta un rango de valores entre 0 "estatus malo" a 1 "estatus alto". Las estimaciones de M-AMBI también siguieron los criterios (i y ii) arriba señalados para AMBI. Finalmente, los valores estimados para AMBI-1 y 2 (como GE), fueron incorporados mediante colores indicativos a los análisis nMDS obtenidos para la abundancia de la macrofauna, esto con el objetivo de visualizar si las agrupaciones comunitarias por factor de interés (e.g., tamiz y periodo) presentaban similares diferencias de acuerdo a los GE.

${ }^{6}<$ http://ambi.azti.es $>$ 
Tabla 2. Listado de taxa identificados en muestras submareales obtenidas en la zona costera oriental del Golfo de Arauco. Se señala el Grupo Ecológico (GE) al cual cada taxon fue asignado de acuerdo al AMBI. Para los taxa categorizados inicialmente como no asignados (N.A.), se señala el mejor y peor GE al cual fueron posteriormente asignados. Números romanos I a $\mathrm{V}$ corresponden a los cinco GE utilizados por AMBI. También se señala el porcentaje (\%) de las abundancias relativas de cada taxon para el total de muestras obtenidas en tamices de 500 y $1.000 \mu \mathrm{m} /$ List of taxa identified in subtidal samples collected in the eastern coastal zone of the Gulf of Arauco. The Ecological Group (GE) to which each taxon was assigned according to $\mathrm{AMBI}$ is indicated. For the taxa initially categorized as not assigned (N.A.), the best and worst GE to which they were later assigned is reported. Roman numerals I to V correspond to the five GE used by AMBI. The percentage (\%) of the relative abundances of each taxon is also shown for the total of samples obtained in 500 and $1,000 \mu \mathrm{m}$ sieves

\begin{tabular}{|c|c|c|c|c|c|}
\hline Taxón & GE AMBI & $\begin{array}{c}\text { Menor } \\
\text { GE }\end{array}$ & $\begin{array}{c}\text { Mayor } \\
\text { GE }\end{array}$ & $\begin{array}{c}\% \mathrm{Ab} \\
500 \mu \mathrm{m}\end{array}$ & $\begin{array}{c}\% \mathrm{Ab} \\
1000 \mu \mathrm{m}\end{array}$ \\
\hline \multicolumn{6}{|l|}{ Annelida } \\
\hline \multicolumn{6}{|l|}{ Polychaeta } \\
\hline \multicolumn{6}{|l|}{ Dorvilleidae } \\
\hline Dorvilleidae sp1 & N.A. & II & V & 0,01 & 0 \\
\hline \multicolumn{6}{|l|}{ Lumbrineridae } \\
\hline Lumbrineris sp. & II & & & 3,48 & 6,86 \\
\hline \multicolumn{6}{|l|}{ Onuphidae } \\
\hline Diopatra sp. & N.A. & I & II & 6,78 & 14,24 \\
\hline Onuphidae sp1 & II & & & 0,04 & 0,06 \\
\hline \multicolumn{6}{|l|}{ Glyceridae } \\
\hline Glycera sp. & II & & & 0,59 & 1,07 \\
\hline \multicolumn{6}{|l|}{ Goniadidae } \\
\hline Goniadidae sp 1 & N.A. & II & II & 0,28 & 0,55 \\
\hline \multicolumn{6}{|l|}{ Nephthyidae } \\
\hline Aglaophamus sp. & II & & & 0,16 & 0 \\
\hline Nephtys sp. & II & & & 0,78 & 1,59 \\
\hline \multicolumn{6}{|l|}{ Nereididae } \\
\hline Nereis sp. & III & & & 0,10 & 0,18 \\
\hline \multicolumn{6}{|l|}{ Phyllodocidae } \\
\hline Eteone sp. & III & & & 0,36 & 0,18 \\
\hline Eumida sp. & II & & & 0,13 & 0,18 \\
\hline Phyllodocidae sp1 & N.A. & II & IV & 0,04 & 0 \\
\hline \multicolumn{6}{|l|}{ Pilargidae } \\
\hline Sigambra sp. & N.A. & II & IV & 0,10 & 0,18 \\
\hline Pilargidae spl & N.A. & I & II & 0,16 & 0,30 \\
\hline \multicolumn{6}{|l|}{ Polynoidae } \\
\hline Polynoidae sp1 & N.A. & II & II & 0,10 & 0,21 \\
\hline \multicolumn{6}{|l|}{ Sigalionidae } \\
\hline Sigalionidae sp1 & N.A. & I & III & 0,43 & 0,49 \\
\hline \multicolumn{6}{|l|}{ Syllidae } \\
\hline Syllidae spl & N.A. & II & II & 0,55 & 0,18 \\
\hline Spionidae & & & & & \\
\hline Boccardia sp. & N.A. & I & IV & 0,03 & 0 \\
\hline Carazziella sp. & III & & & 10,10 & 2,53 \\
\hline Dipolydora sp. & IV & & & 0,01 & 0,03 \\
\hline Paraprionospio sp. & IV & & & 0,39 & 0,82 \\
\hline Polydora sp. & IV & & & 0,04 & 0,09 \\
\hline Prionospio sp. & N.A. & II & IV & 3,85 & 3,66 \\
\hline Scolelepis sp. & III & & & 0,07 & 0,09 \\
\hline Spiophanes sp. & III & & & 0,14 & 0,12 \\
\hline Ampharetidae & & & & & \\
\hline Ampharetidae spl & N.A. & I & III & 0,22 & 0,40 \\
\hline Cirratulidae & & & & & \\
\hline Cirratulidae spl & IV & & & 4,68 & 5,64 \\
\hline Pectinariidae & & & & & \\
\hline Pectinaria sp. & I & & & 0,03 & 0,06 \\
\hline Terebellidae & & & & & \\
\hline Terebellidae spl & N.A. & I & IV & 0,04 & 0,06 \\
\hline Capitellidae & & & & & \\
\hline Mediomastus sp. & III & & & 22,71 & 24,92 \\
\hline Chaetopteridae & & & & & \\
\hline Chaetopteridae spl & N.A. & I & I & 0,07 & 0,12 \\
\hline Cossuridae & & & & & \\
\hline Cossura sp. & IV & & & 1,03 & 1,07 \\
\hline Magelonidae & & & & & \\
\hline Magelona sp. & I & & & 3,14 & 6,25 \\
\hline Orbiniidae & & & & & \\
\hline Orbiniidae sp1 & N.A. & I & I & 0,39 & 0,73 \\
\hline Oweniidae & & & & & \\
\hline Owenia sp. & I & & & 0,12 & 0,24 \\
\hline Paraonidae & & & & & \\
\hline Aricidea sp. & N.A. & I & IV & 5,65 & 2,81 \\
\hline Polygordiidae & & & & & \\
\hline Polygordius sp. & I & & & 3,85 & 2,10 \\
\hline
\end{tabular}

\begin{tabular}{|c|c|c|c|c|c|}
\hline Taxón & GE AMBI & $\begin{array}{c}\text { Menor } \\
\text { GE }\end{array}$ & $\begin{array}{c}\text { Mayor } \\
\text { GE }\end{array}$ & $\begin{array}{c}\% \mathrm{Ab} \\
500 \mu \mathrm{m}\end{array}$ & $\begin{array}{c}\% \mathrm{Ab} \\
1000 \mu \mathrm{m}\end{array}$ \\
\hline \multicolumn{6}{|l|}{ Arthropoda } \\
\hline \multicolumn{6}{|l|}{ Malacostraca } \\
\hline \multicolumn{6}{|l|}{ Ampeliscidae } \\
\hline Ampelisca sp. & I & & & 0,07 & 0,12 \\
\hline Amphipoda sp1 & N.A. & I & II & 0,25 & 0,37 \\
\hline \multicolumn{6}{|l|}{ Alpheidae } \\
\hline Betaeus sp. & I & & & 0,04 & 0,09 \\
\hline \multicolumn{6}{|l|}{ Atelecyclidae } \\
\hline Pseudocorystes sp. & Ignorar & Ignorar & Ignorar & 0,13 & 0,27 \\
\hline \multicolumn{6}{|l|}{ Cancridae } \\
\hline Cancer sp. & N.A. & II & III & 0,14 & 0,30 \\
\hline \multicolumn{6}{|l|}{ Paguridae } \\
\hline Pagurus sp. & II & & & 0,07 & 0,15 \\
\hline \multicolumn{6}{|l|}{ Pinotheridae } \\
\hline Pinnixa sp. & I & & & 0,39 & 0,82 \\
\hline Decapoda spl & Ignorar & Ignorar & Ignorar & 0,04 & 0,09 \\
\hline Isopoda sp1 & Ignorar & Ignorar & Ignorar & 0,09 & 0,15 \\
\hline \multicolumn{6}{|l|}{ Mollusca } \\
\hline \multicolumn{6}{|l|}{ Bivalvia } \\
\hline \multicolumn{6}{|l|}{ Mytilidae } \\
\hline Mytilidae spl & N.A. & III & III & 0,48 & 0,06 \\
\hline \multicolumn{6}{|l|}{ Malletiidae } \\
\hline Malletia sp. & N.A. & I & III & 0,10 & 0,21 \\
\hline \multicolumn{6}{|l|}{ Mactridae } \\
\hline Mulinia sp. & N.A. & III & IV & 0,72 & 1,10 \\
\hline Bivalvia larva & Ignorar & Ignorar & Ignorar & 0,12 & 0,12 \\
\hline \multicolumn{6}{|l|}{ Gastropoda } \\
\hline \multicolumn{6}{|l|}{ Caecidae } \\
\hline Caecidae sp1 & N.A. & I & II & 0,06 & 0 \\
\hline \multicolumn{6}{|l|}{ Nassariidae } \\
\hline Nassarius sp. & II & & & 0,46 & 0,98 \\
\hline Omalogyridae & & & & & \\
\hline Omalogyridae spl & N.A. & III & III & 0,33 & 0,15 \\
\hline Echinodermata & & & & & \\
\hline Ophiuroidea & & & & & \\
\hline Ophiactidae & & & & & \\
\hline Ophiactis sp. & II & & & 0,13 & 0,27 \\
\hline Ophiurida sp1 & II & & & 5,81 & 10,73 \\
\hline Chordata & & & & & \\
\hline Actinopterygii & & & & & \\
\hline Agonidae & & & & & \\
\hline Agonopsis chiloensis & Ignorar & Ignorar & Ignorar & 0,01 & 0,03 \\
\hline Cnidaria & & & & & \\
\hline Anthozoa & & & & & \\
\hline Actiniaria sp1 & II & & & 0,36 & 0,76 \\
\hline Hemichordata & & & & & \\
\hline Enteropneusta & & & & & \\
\hline Harrimaniidae & & & & & \\
\hline Saccoglossus sp. & I & & & 0,01 & 0,03 \\
\hline Sipuncula & & & & & \\
\hline Sipunculidae & & & & & \\
\hline Sipunculidae spl & N.A. & I & I & 0,01 & 0,03 \\
\hline Nemertea & & & & & \\
\hline Nemertea spp. & III & & & 0,93 & 1,86 \\
\hline Nematoda & & & & & \\
\hline Nematoda spp. & III & & & 17,66 & 2,87 \\
\hline Foraminifera & & & & & \\
\hline Foraminifera spp. & Ignorar & Ignorar & Ignorar & 0,87 & 0,37 \\
\hline $\begin{array}{l}\text { Taxonomía de acu } \\
\text { (http://www.marin }\end{array}$ & $\begin{array}{l}\text { lo al World } \\
\text { pecies.org) }\end{array}$ & egister of & arine Spe & & \\
\hline
\end{tabular}




\section{RESUltados}

Para la macrofauna identificada en toda la zona de estudio, el total de taxa incluyendo las 5 áreas y ambos períodos de muestreo fue de 62 (Tabla 2). Se registró 51 y 47 taxa $(500$ y $1.000 \mu \mathrm{m})$ en invierno y, 54 y $51 \operatorname{taxa}(500 \mathrm{y}$ $1.000 \mu \mathrm{m})$ en verano. Los análisis PERMANOVA para la riqueza de especies/taxa, equidad de Pielou, diversidad de Shannon-Wiener y dominancia de Simpson entregaron diferencias significativas entre tamices $(P$ (perm $)<0,05$; Tabla 3). En general, en el tamiz de $500 \mu \mathrm{m}$ se observó una mayor riqueza de especies (4 taxa en promedio), mayor diversidad Shannon-Wiener (0,1 unidad en promedio) y, menor dominancia Simpson ( 0,2 unidades en promedio) y equidad Pielou (0,4 unidades en promedio) (Fig. 1). Este patrón se repite para las 5 áreas y en ambos períodos de muestreo, representados gráficamente para la riqueza de especies/taxa (Fig. 2a, b). Respecto a la abundancia total, si bien el análisis PERMANOVA no detecta diferencias significativas entre tamices (Tabla 3), probablemente asociado a la alta variabilidad de las abundancias entre muestras, en el tamiz de $500 \mu \mathrm{m}$ se observó siempre valores promedio casi del doble respecto al tamiz de $1.000 \mu \mathrm{m}$ (Figs. 1 y 2). Cuantitativamente, de los 10.182 individuos contabilizados, en el tamiz de $1.000 \mu \mathrm{m}$ se registraron sólo 3.279 , lo que corresponde a un $67,8 \%$ de pérdida en abundancia total respecto al tamiz de $500 \mu \mathrm{m}$. a)

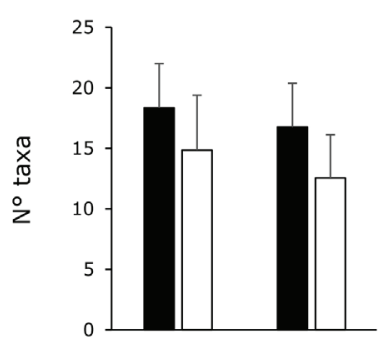

c)

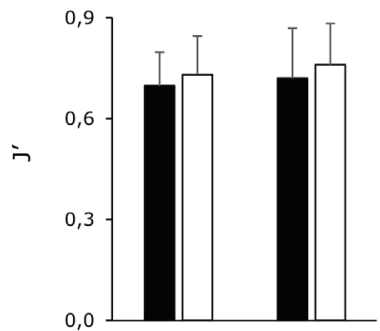

e)

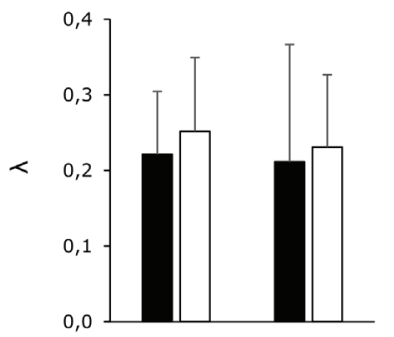

g)

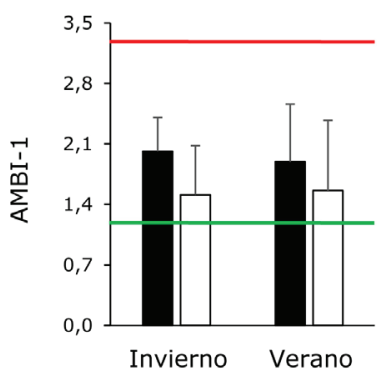

b)

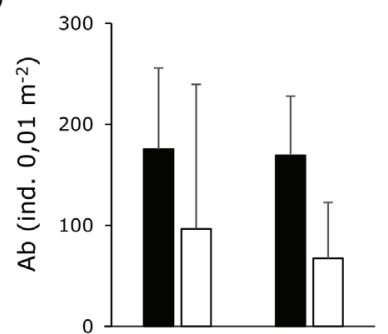

d)

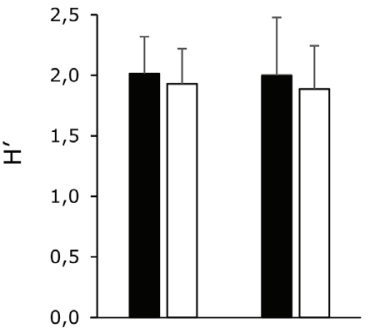

f)

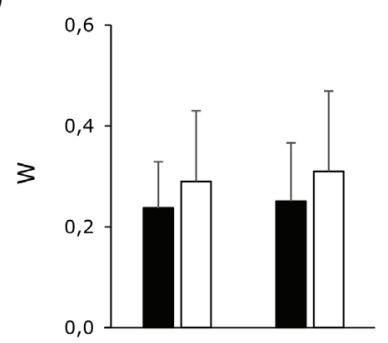

h)

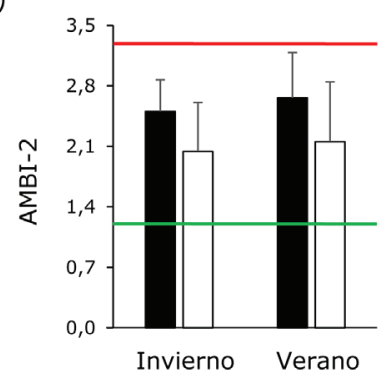

Figura 1. Valores promedio ( $\pm D E$, barras de error) para muestras de macrofauna marina bentónica obtenidas mediante tamices de $500 \mu \mathrm{m}$ (negro) y $1.000 \mu \mathrm{m}$ (blanco) en períodos de invierno y verano en el Golfo de Arauco. a) Riqueza de especies/taxa, b) Abundancia total, c) Equidad Pielou, d) Diversidad Shannon-Wiener, e) Dominancia Simpson, f) Índice de Warwick, g) Índice AMBI-1 y, h) Índice AMBI-2. Las líneas verdes y rojas representan el límite superior de las categorías no-contaminado y levemente contaminado de AMBI / Mean values ( \pm SD, error bars) for samples of benthic marine macrofauna obtained using sieves of $500 \mu \mathrm{m}$ (black) and 1,000 $\mu \mathrm{m}$ (white) in winter and summer in the Gulf of Arauco. a) Species/ taxa richness, b) Total abundance, c) Pielou's evenness, d) Shannon-Wiener diversity, e) Simpson's dominance, f) Warwick index, g) AMBI-1 index and, h) AMBI-2 index. Green and red lines represent the upper limit of no-polluted and slightly polluted categories according to AMBI 
De acuerdo al análisis SIMPER, del total de taxa identificados, considerando las 5 áreas para los dos periodos de muestreo, los 5 taxa en promedio más abundantes (ind. 0,01 $\mathrm{m}^{-2}$ ), y expresados en porcentaje, en el tamiz de $500 \mu \mathrm{m}$ fueron Mediomastus sp. $(39,20)$, Nematoda $(30,48)$, Carazziella sp. $(17,43)$, Diopatra sp. $(11,70)$ y Ophiuroidea $(10,03)$; mientras que, en el tamiz de $1.000 \mu \mathrm{m}$ fueron Mediomastus sp. $(20,43)$, Diopatra sp. $(11,68)$, Ophiuroidea $(8,80)$, Lumbrineris sp. $(5,63)$ y Magelona sp. $(5,13)$ (Tabla 2). La mayor disimilitud porcentual entre tamices en términos de sus abundancias estuvo dada por Mediomastus sp. (18,89\%), Nematoda (13,09\%), Carazziella sp. (9,06\%), Diopatra sp. (8,13\%) y Ophiuroidea (8,12\%). En términos de la biomasa (g 0,01 $\mathrm{m}^{-2}$ ) en promedio los 3 taxa más representativos en el tamiz de $500 \mu \mathrm{m}$ fueron Diopatra sp. (3,060), Nassarius sp. (1,260) y Lumbrineris sp. (0,390); mientras que, en el tamiz de $1.000 \mu \mathrm{m}$ fueron Mediomastus sp. (0,003 g), Carazziella sp. $(0,002)$ y Aricidea sp. (0,001). La mayor disimilitud porcentual entre tamices en términos de sus biomasas estuvo dada por Diopatra sp. (39,38\%), Nassarius sp. (21,26\%) y Lumbrineris sp. (7,89\%). a)

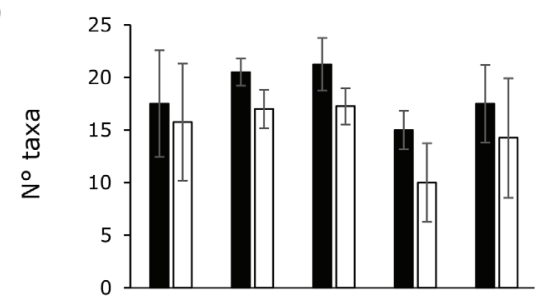

c)

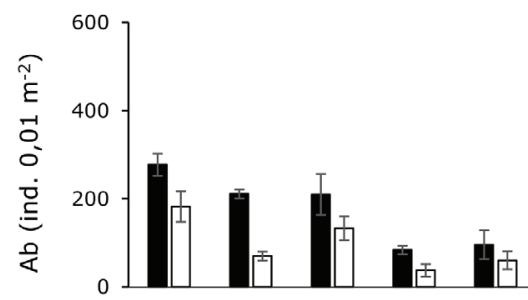

e)

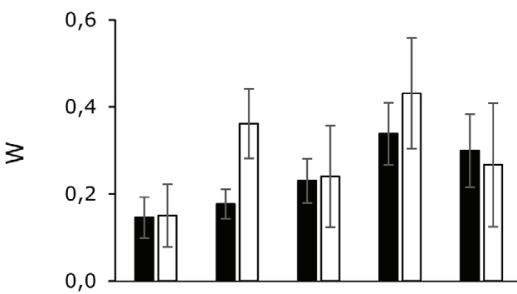

g)

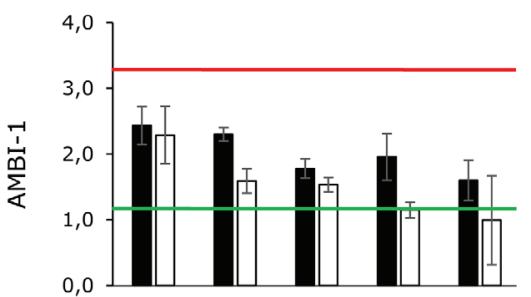

i)

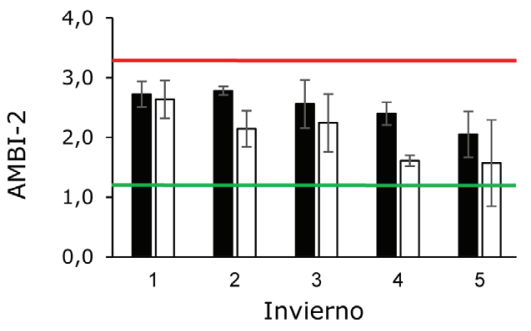

b)

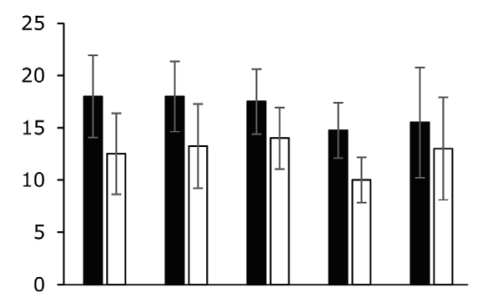

d)

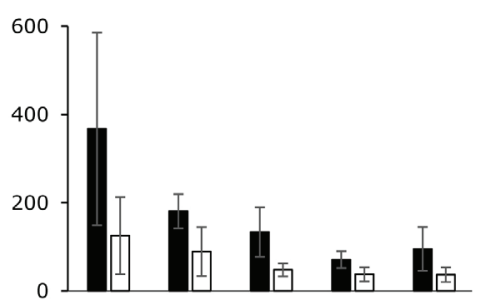

f)

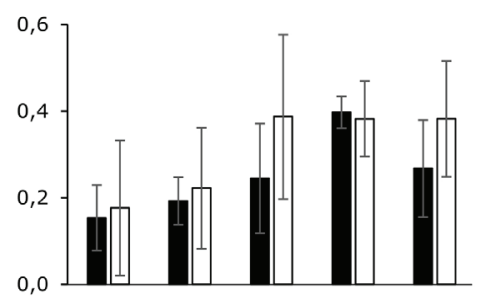

h)

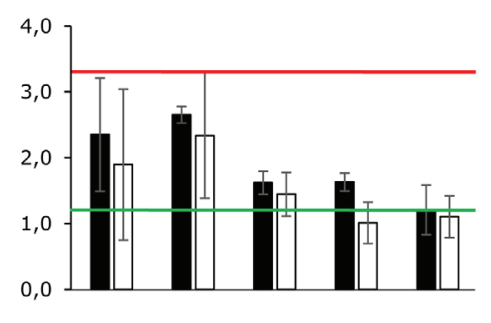

j)

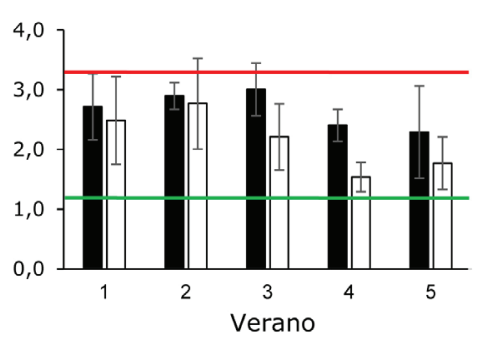

Figura 2. Valores promedio ( $\pm \mathrm{DE}$, barras de error) para muestras de macrofauna marina bentónica obtenidas mediante tamices de $500 \mu \mathrm{m}$ (negro) y $1.000 \mu \mathrm{m}$ (blanco) en períodos de invierno y verano en 5 áreas de muestreo en el Golfo de Arauco. a-b) Riqueza de especies/taxa, c-d) Abundancia total, e-f) Índice Warwick, g-h) Índice AMBI-1 y, i-j) Índice AMBI-2. Las líneas verdes y rojas representan el límite superior de las categorías nocontaminado y levemente contaminado de AMBI / Mean values ( \pm SD, error bars) for samples of benthic marine macrofauna obtained using sieves of $500 \mu \mathrm{m}$ (black) and 1,000 $\mu \mathrm{m}$ (white) in winter and summer in 5 areas of the Gulf of Arauco. a-b) Species/taxa richness, c-d) Total abundance, e-f) Warwick index, g-h) AMBI-1 index, and i-j) AMBI-2 index. Green and red lines represent the upper limit of no-polluted and slightly polluted categories according to $\mathrm{AMBI}$ 
Tabla 3. Análisis PERMANOVA para las variables comunitarias univariadas de la macrofauna marina bentónica obtenida en el Golfo de Arauco. Los factores de análisis corresponden a: Período (invierno y verano) y Tamiz (500 y $1.000 \mu \mathrm{m})$. En negrita valores $P$ (perm) $<0,05 /$ PERMANOVA analysis for the measured univariate community variables of the marine benthic macrofauna obtained in the Gulf of Arauco. The factors used in the analysis were Period (winter and summer) and sieve size (500 and 1,000 $\mu \mathrm{m})$. Values of $P$ (perm) $<0.05$ are shown in bold

\begin{tabular}{|c|c|c|c|c|c|c|}
\hline Fuente & g.l. & SCE & $\mathrm{CME}$ & Pseudo-F & $\begin{array}{c}P \\
\text { (perm) }\end{array}$ & $\begin{array}{l}\text { Perm. } \\
\text { únicas }\end{array}$ \\
\hline \multicolumn{7}{|l|}{ Riqueza de especies } \\
\hline Periodo & 1 & 23,113 & 23,113 & 1,8907 & 0,187 & 992 \\
\hline Tamiz & 1 & 556,51 & 556,51 & 45,525 & 0,001 & 991 \\
\hline Período $\times$ Tamiz & 1 & 30,013 & 30,013 & 2,4551 & 0,125 & 997 \\
\hline Residuos & 76 & 929,05 & 12,224 & & & \\
\hline Total & 79 & 1538,7 & & & & \\
\hline \multicolumn{7}{|l|}{ Abundancia total } \\
\hline Período & 1 & 177,01 & 177,01 & 0,038392 & 0,861 & 995 \\
\hline Tamiz & 1 & 1487,8 & 1487,8 & 0,32269 & 0,538 & 998 \\
\hline Periodo x Tamiz & 1 & 13913 & 13913 & 3,0176 & 0,104 & 991 \\
\hline Residuos & 76 & $3,50 \mathrm{E}+05$ & 4610,6 & & & \\
\hline Total & 79 & $3,66 \mathrm{E}+05$ & & & & \\
\hline \multicolumn{7}{|l|}{ Equidad de Pielou } \\
\hline Período & 1 & 0,057293 & 0,057293 & 3,3157 & 0,077 & 995 \\
\hline Tamiz & 1 & 0,090391 & 0,090391 & 5,2312 & 0,030 & 997 \\
\hline Período x Tamiz & 1 & 0,011417 & 0,011417 & 0,66074 & 0,397 & 994 \\
\hline Residuos & 76 & 1,3132 & 0,017279 & & & \\
\hline Total & 79 & 1,4723 & & & & \\
\hline \multicolumn{7}{|c|}{ Diversidad Shannon-Wiener } \\
\hline Período & 1 & 0,080056 & 0,080056 & 0,51828 & 0,469 & 998 \\
\hline Tamiz & 1 & 5,2296 & 5,2296 & 33,857 & 0,001 & 998 \\
\hline Período x Tamiz & 1 & 0,22586 & 0,22586 & 1,4622 & 0,246 & 995 \\
\hline Residuos & 76 & 11,739 & 0,15446 & & & \\
\hline Total & 79 & 17,275 & & & & \\
\hline \multicolumn{7}{|c|}{ Dominancia de Simpson } \\
\hline Período & 1 & 0,064428 & 0,064428 & 3,4801 & 0,073 & 995 \\
\hline Tamiz & 1 & 0,26409 & 0,26409 & 14,264 & 0,001 & 995 \\
\hline Período $x$ Tamiz & 1 & 0,025615 & 0,025615 & 1,3836 & 0,256 & 999 \\
\hline Residuos & 76 & 1,407 & 0,018514 & & & \\
\hline Total & 79 & 1,7612 & & & & \\
\hline \multicolumn{7}{|l|}{ Índice Warwick } \\
\hline Período & 1 & 0,094395 & 0,094395 & 1,8648 & 0,177 & 993 \\
\hline Tamiz & 1 & 1,2888 & 1,2888 & 25,462 & 0,001 & 995 \\
\hline Período $\times$ Tamiz & 1 & 0,047556 & 0,047556 & 0,9395 & 0,356 & 998 \\
\hline Residuos & 76 & 3,847 & 0,050618 & & & \\
\hline Total & 79 & 5,2778 & & & & \\
\hline \multicolumn{7}{|l|}{ Índice AMBI-1 } \\
\hline Período & 1 & 0,43498 & 0,43498 & 1,1259 & 0,302 & 997 \\
\hline Tamiz & 1 & 13,505 & 13,505 & 34,956 & 0,001 & 997 \\
\hline Período x Tamiz & 1 & 0,77126 & 0,77126 & 1,9964 & 0,167 & 998 \\
\hline Residuos & 76 & 29,361 & 0,38633 & & & \\
\hline Total & 79 & 44,072 & & & & \\
\hline \multicolumn{7}{|l|}{ Índice AMBI-2 } \\
\hline Período & 1 & 0,2003 & 0,2003 & 0,6697 & 0,412 & 999 \\
\hline Tamiz & 1 & 17,18 & 17,18 & 57,441 & 0,001 & 999 \\
\hline Período $\mathrm{x}$ Tamiz & 1 & 0,0026796 & 0,0026796 & 0,0089593 & 0,899 & 996 \\
\hline Residuos & 76 & 22,731 & 0,29909 & & & \\
\hline Total & 79 & 40,114 & & & & \\
\hline
\end{tabular}

Tabla 4. Análisis PERMANOVA para las abundancias y biomasas de la macrofauna marina bentónica del Golfo de Arauco. Los factores de análisis corresponden a período (invierno y verano) y áreas de muestreo (5 áreas). En negrita valores $P$ (perm) $<0,05$ / PERMANOVA analysis for the abundance and biomass of the marine benthic macrofauna of the Gulf of Arauco. The factors used in the analysis were period (winter and summer) and sampling areas (5 areas). Values of $P($ perm $)<0.05$ are shown in bold

\begin{tabular}{|c|c|c|c|c|c|c|}
\hline Fuente & g.l. & SCE & CME & Pseudo-F & $P($ perm $)$ & $\begin{array}{l}\text { Perm. } \\
\text { únicas }\end{array}$ \\
\hline \multicolumn{7}{|c|}{ Abundancia $(500 \mu \mathrm{m})$} \\
\hline Período & 1 & 3094,8 & 3094,8 & 3,6743 & 0,001 & 997 \\
\hline Área & 4 & 17526 & 4381,4 & 5,2018 & 0,001 & 999 \\
\hline Período x Área & 4 & 6021,1 & 1505,3 & 1,7871 & 0,003 & 997 \\
\hline Residuos & 30 & 25269 & 842,29 & & & \\
\hline Total & 39 & 51910 & & & & \\
\hline \multicolumn{7}{|c|}{ Abundancia $(1.000 \mu \mathrm{m})$} \\
\hline Período & 1 & 3345,5 & 3345,5 & 2,2724 & 0,010 & 999 \\
\hline Área & 4 & 21069 & 5267,2 & 3,5776 & 0,001 & 999 \\
\hline Período x Área & 4 & 7511,3 & 1877,8 & 1,2755 & 0,118 & 998 \\
\hline Residuos & 30 & 44168 & 1472,3 & & & \\
\hline Total & 39 & 76094 & & & & \\
\hline \multicolumn{7}{|l|}{ Biomasa $(500 \mu \mathrm{m})$} \\
\hline Período & 1 & 3515,5 & 3515,5 & 2,3064 & 0,013 & 998 \\
\hline Área & 4 & 17181 & 4295,3 & 2,818 & 0,001 & 999 \\
\hline Período x Área & 4 & 8645,1 & 2161,3 & 1,4179 & 0,073 & 997 \\
\hline Residuos & 30 & 45727 & 1524,2 & & & \\
\hline Total & 39 & 75069 & & & & \\
\hline \multicolumn{7}{|l|}{ Biomasa $(1.000 \mu \mathrm{m})$} \\
\hline Período & 1 & 3304,9 & 3304,9 & 1,8599 & 0,060 & 998 \\
\hline Área & 4 & 18600 & 4649,9 & 2,6169 & 0,001 & 998 \\
\hline Período x Área & 4 & 9358,8 & 2339,7 & 1,3167 & 0,123 & 998 \\
\hline Residuos & 30 & 53307 & 1776,9 & & & \\
\hline Total & 39 & 84570 & & & & \\
\hline
\end{tabular}

En relación a la disimilitud comunitaria multivariada, tanto para las abundancias como para las biomasas, el análisis PERMANOVA, realizado por separado para cada tamiz, encontró diferencias significativas entre muestreos $(P$ (perm) $<0,1$, Tabla 4). Para las áreas de muestreo, el análisis PERMANOVA también detectó diferencias significativas en las abundancias y biomasas para ambos tamices por separado $(P$ (perm) $<0,05$, Tabla 4$)$. Los análisis a posteriori pair-wise entregaron diferencias significativas $(P($ perm $)<0,05)$ entre las 5 áreas de muestreos para los análisis de abundancia y biomasa realizados (Tabla 4). Para las abundancias en $500 \mu \mathrm{m}$ se presentó además una interacción significativa entre área y período de muestreo $(P$ (perm) $<0,05$; Tabla 4$)$. Gráficamente, las disimilitudes comunitarias multivariadas detectadas para ambos factores y para cada tamiz por separado son presentadas en análisis mMDS (Figs. 3 y 4). 
a)

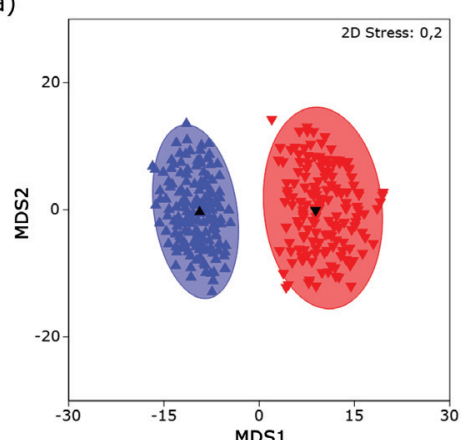

c)

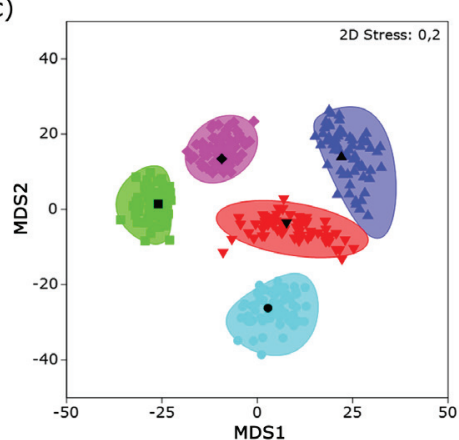

b)

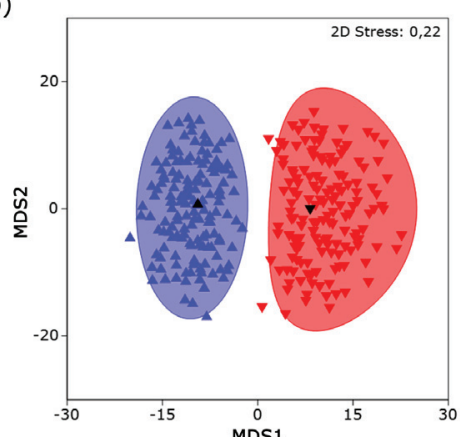

d)

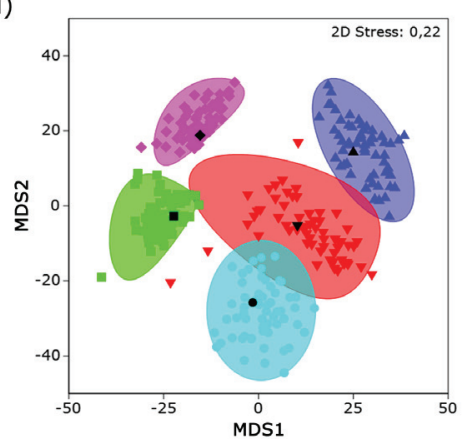

Figura 3. Análisis métrico de escalamiento multidimensional (mMDS) para la abundancia de la macrofauna marina bentónica obtenida mediante tamices de $500 \mu \mathrm{m}(\mathrm{a}, \mathrm{c})$ y $1.000 \mu \mathrm{m}$ (b, d) en 5 áreas de muestreo en el Golfo de Arauco. En el panel de arriba se presentan los pseudo-intervalos de confianza al $95 \%$ para los muestreos de invierno (elipse azul) y verano (elipse roja). En el panel de abajo se representan en diferentes colores los pseudo-intervalos de confianza al $95 \%$ para las 5 áreas de muestreo. Símbolos en negro representan a los centroides estimados para cada factor y nivel de análisis / Metric multidimensional scaling (mMDS) analysis for abundances of marine benthic macrofauna obtained using sieves of $500 \mu \mathrm{m}$ $(a, c)$ and $1,000 \mu \mathrm{m}(b, d)$ in 5 areas of the Gulf of Arauco. The upper panel shows the $95 \%$ pseudo-confidence limits for the winter (blue ellipse) and summer (red ellipse). The lower panel presents the $95 \%$ pseudo-confidence intervals for the 5 sampled areas. Black symbols indicate the centroids estimated for each factor and level of analysis

a)

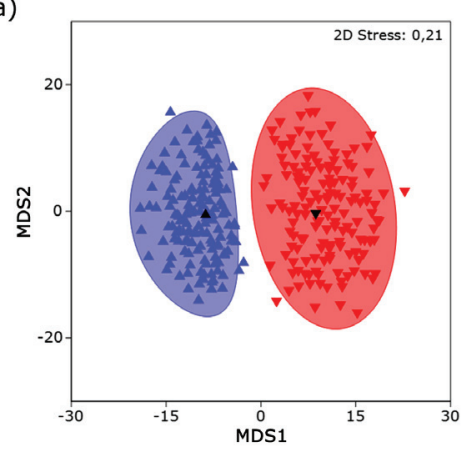

c)

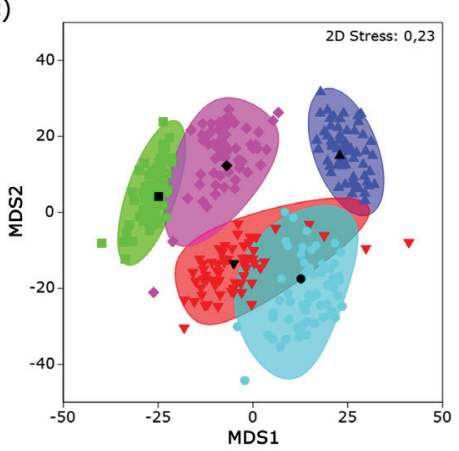

b)

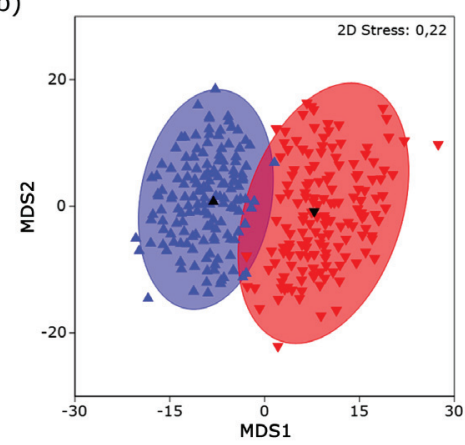

d)

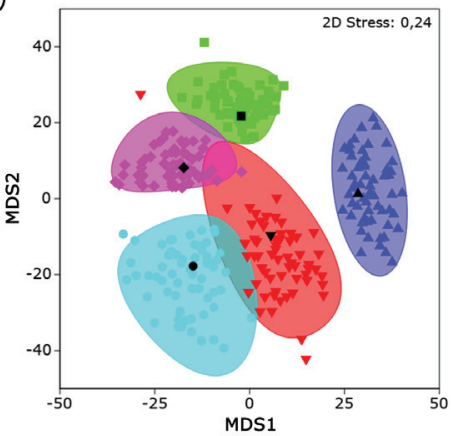

Figura 4. Análisis métrico de escalamiento multidimensional (mMDS) para la biomasa de la macrofauna marina bentónica obtenida mediante tamices de $500 \mu \mathrm{m}(\mathrm{a}, \mathrm{c})$ y $1.000 \mu \mathrm{m}$ (b, d) en 5 áreas de muestreo en el Golfo de Arauco. En el panel de arriba se presentan los pseudo-intervalos de confianza al $95 \%$ para los muestreos de invierno (elipse azul) y verano (elipse roja). En el panel de abajo se representan en diferentes colores los pseudo-intervalos de confianza al $95 \%$ para las 5 áreas de muestreo. Símbolos en negro representan a los centroides estimados para cada factor y nivel de análisis / Metric multidimensional scaling (mMDS) analysis for biomass of marine benthic macrofauna obtained using sieves of $500 \mu \mathrm{m}$ $(a, c)$ and 1,000 $\mu \mathrm{m}(b, d)$ in 5 areas of the Gulf of Arauco. The upper panel shows the $95 \%$ pseudo-confidence limits for the winter (blue ellipse) and summer (red ellipse). The lower panel presents the $95 \%$ pseudo-confidence intervals for the 5 sampled areas. Black symbols indicate the centroids estimated for each factor and level of analysis 
Al comparar la disimilitud comunitaria multivariada entre tamices, tanto para las abundancias como para las biomasas, el análisis PERMANOVA entregó diferencias significativas $(P$ (perm) $<0,05$; Tabla 5$)$. También se presentaron diferencias significativas $(P($ perm $)<0,05)$, tanto para las abundancias como para las biomasas, entre períodos y áreas de muestreo (Tabla 5). Los análisis a posteriori pair-wise entregan diferencias significativas $(P($ perm $)<0,05)$ para las abundancias y biomasas entre las 5 áreas de muestreo. Para las abundancias y biomasas se presentó además una interacción significativa entre área y período; mientras que para las biomasas además hubo una interacción significativa entre área y tamiz $(P$ (perm) $<0,05$; Tabla 5). Las disimilitudes comunitarias multivariadas entre tamices pueden observarse en los análisis nMDS y mMDS (Fig. 5) y en los análisis PCO (Figs. 6 y 7), permitiendo este último análisis cuantificar porcentualmente estas disimilitudes para las 5 áreas de muestreo $(63,2$ y $76,6 \%$ de variación total explicada para las abundancias y biomasas, respectivamente) y, entre invierno y verano (100 y 98,4\% de variación total explicada para las abundancias y biomasas, respectivamente).

Tabla 5. Análisis PERMANOVA obtenidos para las abundancias y biomasas de la macrofauna marina bentónica en el Golfo de Arauco. Los factores de análisis corresponden a: período (invierno y verano), área (5 áreas) y tamiz (500 y $1.000 \mu \mathrm{m})$. En negrita valores $P$ (perm) $<0,05$ / PERMANOVA analysis for the abundance and biomass of the marine benthic macrofauna of the Gulf of Arauco. The factors used in the analysis were period (winter and summer), sampling area ( 5 area) and sieve size $(500$ and $1,000 \mu \mathrm{m})$. Values of $P($ perm $)<0.05$ are shown in bold

\begin{tabular}{lcccccc}
\hline \multicolumn{1}{c}{ Fuente } & g.l. & SCE & CME & Pseudo-F & $P$ (perm) & $\begin{array}{c}\text { Perm. } \\
\text { únicas }\end{array}$ \\
\hline Abundancia & & & & & & \\
$\quad$ Período & 1 & 6306,9 & 6306,9 & 5,4497 & $\mathbf{0 , 0 0 1}$ & 997 \\
$\quad$ Área & 4 & 37866 & 9466,4 & 8,1799 & $\mathbf{0 , 0 0 1}$ & 998 \\
Tamiz & 1 & 7607,6 & 7607,6 & 6,5737 & $\mathbf{0 , 0 0 1}$ & 998 \\
Período x Área & 4 & 13471 & 3367,8 & 2,9101 & $\mathbf{0 , 0 0 1}$ & 997 \\
Período x Tamiz & 1 & 133,51 & 133,51 & 0,11536 & 0,995 & 999 \\
Área x Tamiz & 4 & 728,74 & 182,19 & 0,15743 & 1 & 998 \\
Período x Área x Tamiz & 4 & 61,286 & 15,322 & 0,013239 & 1 & 998 \\
Residuos & 60 & 69437 & 1157,3 & & & \\
Total & 79 & $1,36 \mathrm{E}+05$ & & & & \\
Biomasa & & & & & & \\
Período & 1 & 7180,3 & 7180,3 & 5,4259 & $\mathbf{0 , 0 0 1}$ & 998 \\
Área & 4 & 30141 & 7535,2 & 5,6941 & $\mathbf{0 , 0 0 1}$ & 998 \\
Tamiz & 1 & 65735 & 65735 & 49,674 & $\mathbf{0 , 0 0 1}$ & 999 \\
Período x Área & 4 & 13206 & 3301,5 & 2,4948 & $\mathbf{0 , 0 0 1}$ & 999 \\
Período x Tamiz & 1 & 1637,8 & 1637,8 & 1,2377 & 0,251 & 997 \\
Área x Tamiz & 4 & 7678,4 & 1919,6 & 1,4506 & $\mathbf{0 , 0 1 8}$ & 999 \\
Período x Área x Tamiz & 4 & 3279,6 & 819,9 & 0,61957 & 0,984 & 997 \\
Residuos & 60 & 79400 & 1323,3 & & & \\
Total & 79 & $2,08 \mathrm{E}+05$ & & & & \\
\hline
\end{tabular}

a)

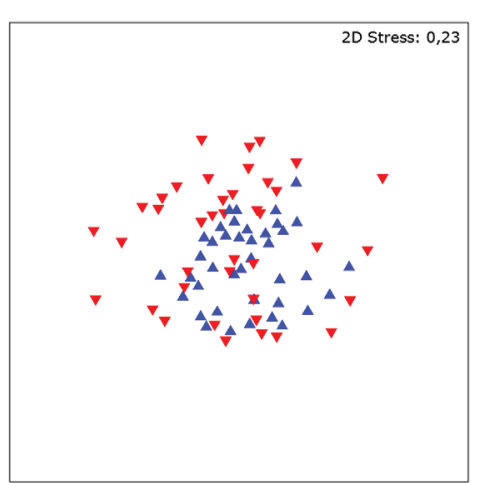

c)

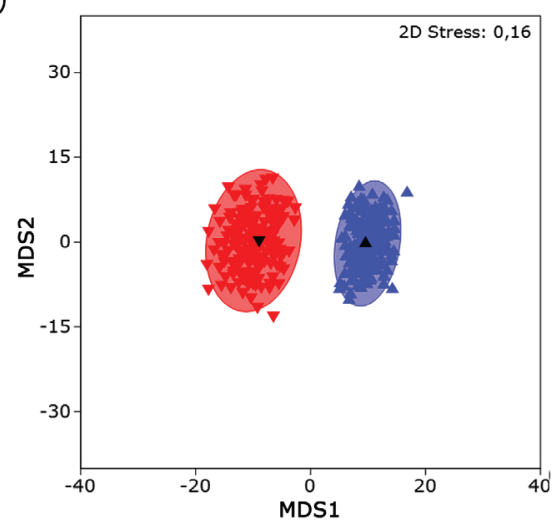

b)

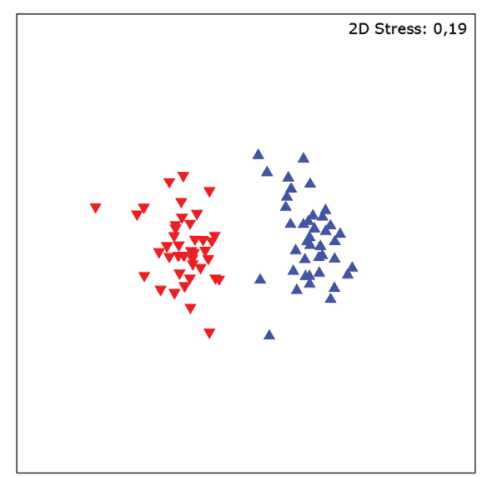

d)

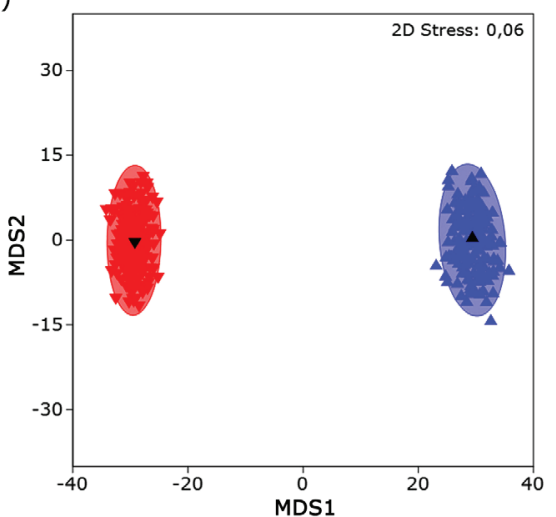

Figura 5. Análisis no-métrico de escalamiento multidimensional (nMDS) (a, b) y métrico de escalamiento multidimensional (mMDS) (c, d), para las abundancias (panel izquierdo) y biomasas (panel derecho) de la macrofauna marina bentónica obtenida en el Golfo de Arauco. Los paneles de la izquierda y derecha entregan los resultados para los tamices de $500 \mu \mathrm{m}$ (azul) y $\mathbf{1 . 0 0 0} \boldsymbol{\mu m}$ (rojo). En (c y d) se presentan mediante elipses los pseudo-intervalos de confianza al 95\% y los centroides señalados con símbolos de color negro / Non-metric (nMDS) (a, b) and metric (mMDS) (c, d) multidimensional scaling analyses for abundance (left panel) and biomass (right panel) of marine benthic macrofauna obtained in the Gulf of Arauco. The left and right panels illustrate the results for sieve sizes of $500 \mu \mathrm{m}$ (blue) and 1,000 $\mu \mathrm{m}$ (red). The $95 \%$ pseudo-confidence intervals are shown in c and d; centroids are the black symbols 
a)

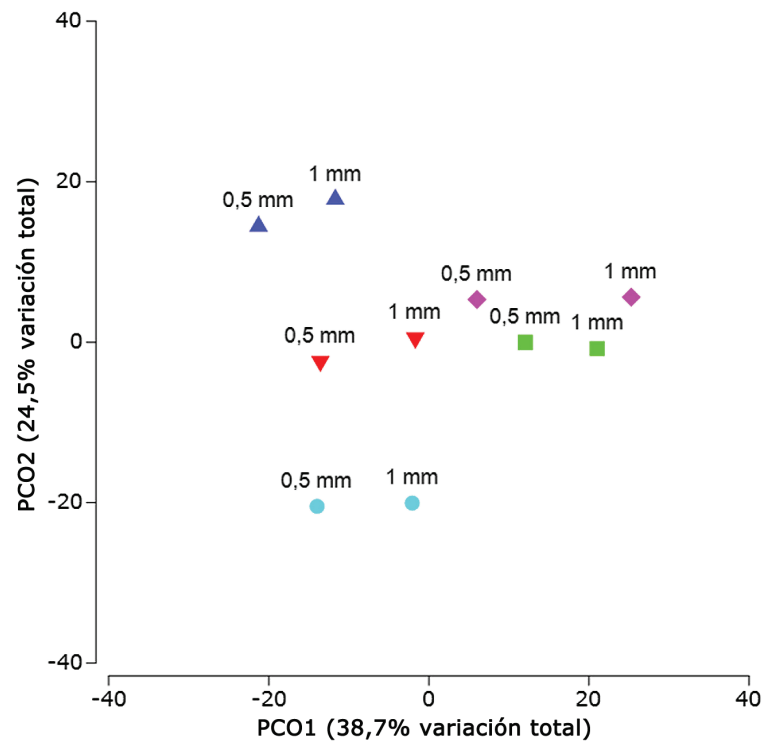

b)

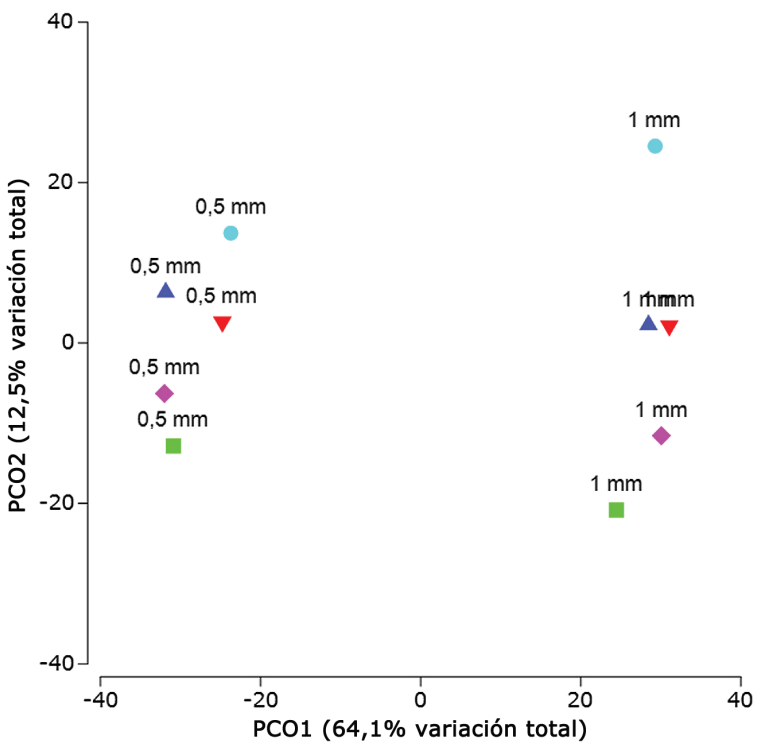

Figura 6. Análisis de ordenación de coordenadas principales (PCO) de la macrofauna marina bentónica del Golfo de Arauco obtenida mediante tamices de $500 \mu \mathrm{m}(0,5 \mathrm{~mm})$ y $1.000 \mu \mathrm{m}(1 \mathrm{~mm})$ para las abundancias (a) y biomasas (b). En ambas gráficas se señala mediante símbolos de colores las 5 áreas de muestreo, etiquetando además el tamaño del tamiz utilizado. Cada gráfica da cuenta del porcentaje total de variación explicado por los ejes PCO1 y PCO2 / Principal coordinates analysis (PCO) of the benthic marine macrofauna of the Gulf of Arauco obtained with sieve sizes of $500 \mu \mathrm{m}(0.5 \mathrm{~mm})$ and 1,000 $\mu \mathrm{m}(1 \mathrm{~mm})$ for abundances (a) and biomass (b). In both graphs the colored symbols indicate the 5 sampling areas; sieve size is also indicated. The graphs show the total percent of variation explained by the PCO1 and PCO2 axes

a)

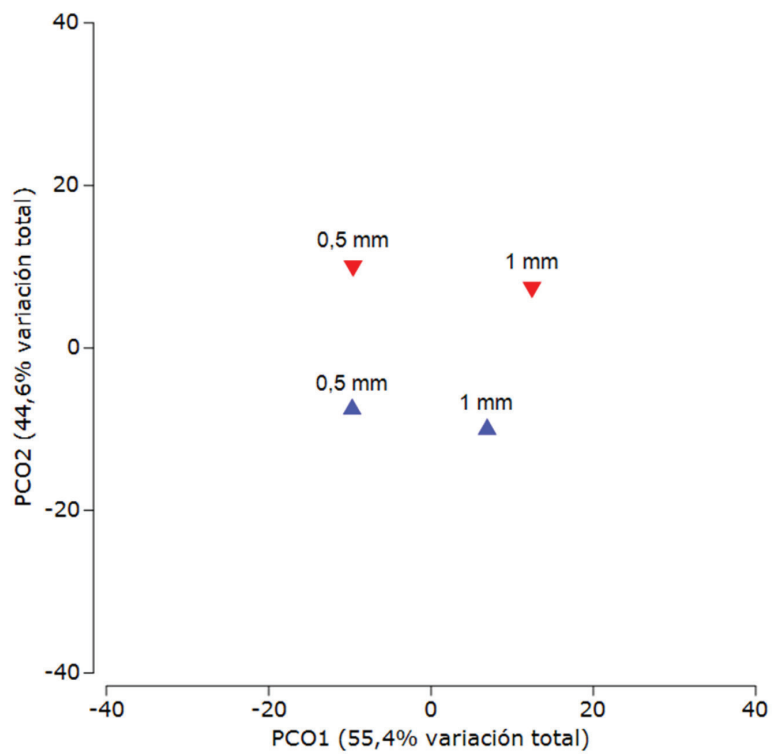

b)

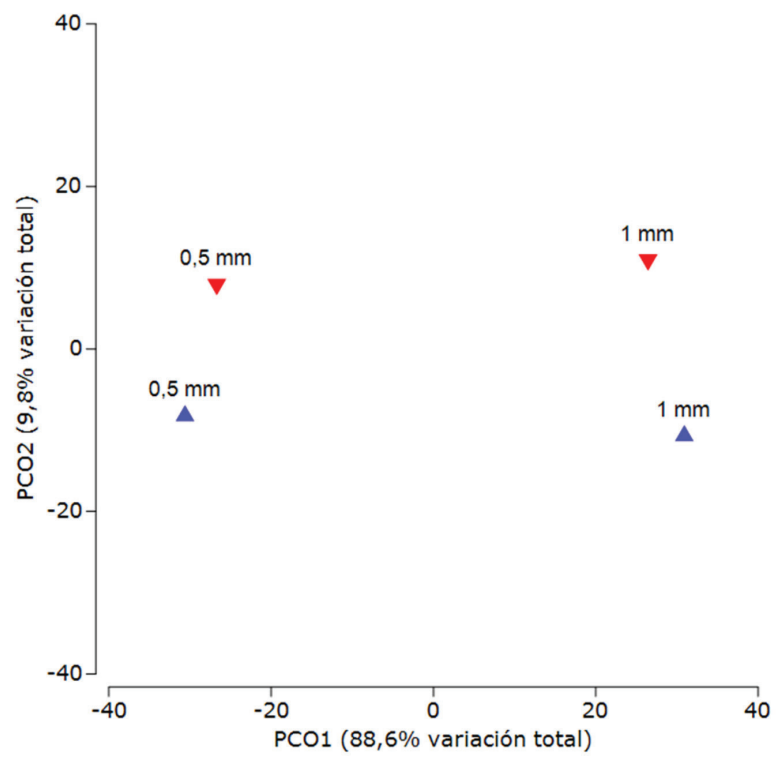

Figura 7. Análisis de ordenación de coordenadas principales (PCO) de la macrofauna marina bentónica del Golfo de Arauco obtenida mediante tamices de $500 \mu \mathrm{m}(0,5 \mathrm{~mm})$ y $1.000 \mu \mathrm{m}(1 \mathrm{~mm})$ para las abundancias (a) y biomasas (b). En ambas gráficas se señala mediante símbolos de colores los dos períodos de muestreo (invierno: azul y verano: rojo), etiquetando además el tamaño del tamiz utilizado. Cada gráfica da cuenta del porcentaje total de variación explicado por los ejes PCO1 y PCO2 / Principal coordinates analysis (PCO) of the benthic marine macrofauna of the Gulf of Arauco obtained with sieve sizes of $500 \mu \mathrm{m}(0.5 \mathrm{~mm})$ and 1,000 $\mu \mathrm{m}(1 \mathrm{~mm})$ for abundances (a) and biomass (b). In both graphs the colored symbols indicate the two sampling periods (winter: blue; summer: red); sieve size is also indicated. The graphs show the total percent of variation explained by the PCO1 and PCO2 axes 
Para los bio-indicadores comunitarios (W y AMBI), los resultados indican que $\mathrm{W}$ presentó valores promedio más bajos en el tamiz de $500 \mu \mathrm{m}$ en invierno y verano ( $P$ (perm) $<0,05$; Tabla 3, Fig. 1f). En el caso de AMBI-1 y 2, ambos estimadores presentaron en invierno y verano valores promedio mayor en el tamiz de $500 \mu \mathrm{m}$, siendo AMBI-2 el que entregó los más altos $(P($ perm $)<0,05$; Tabla 3, Fig. 1g-h). Estos resultados son en general similares cuando los estimadores son observados para cada área de muestreo (Fig. 2e-j). El detalle de los valores obtenidos para AMBI-1 y 2 y los porcentajes de los GE en ambos tamices para cada muestra son presentados en la Figura 8. De acuerdo a los valores estimados para cada muestra, en el caso de AMBI-1 estos se encuentran entre 1,0 y $3,0(500 \mu \mathrm{m})$ y, entre 0,5 y

a)

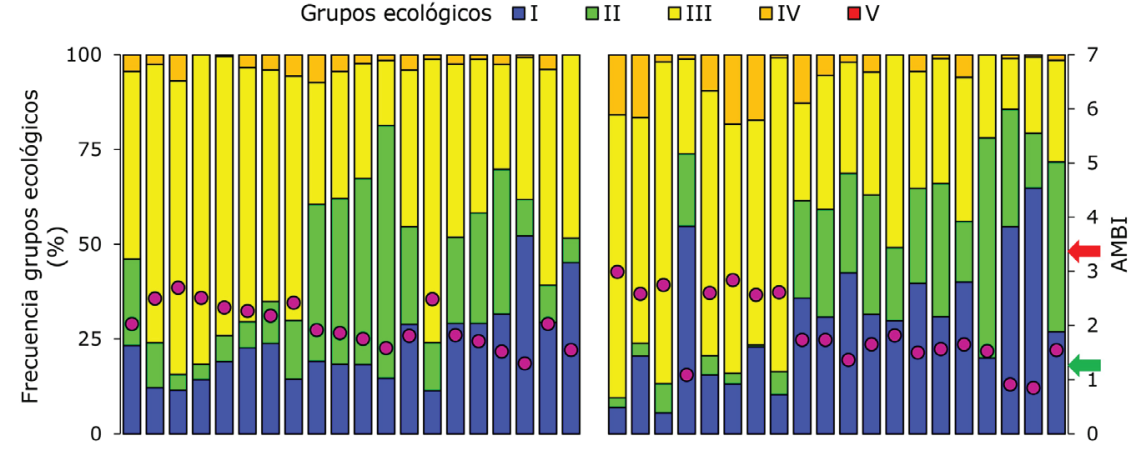

b)

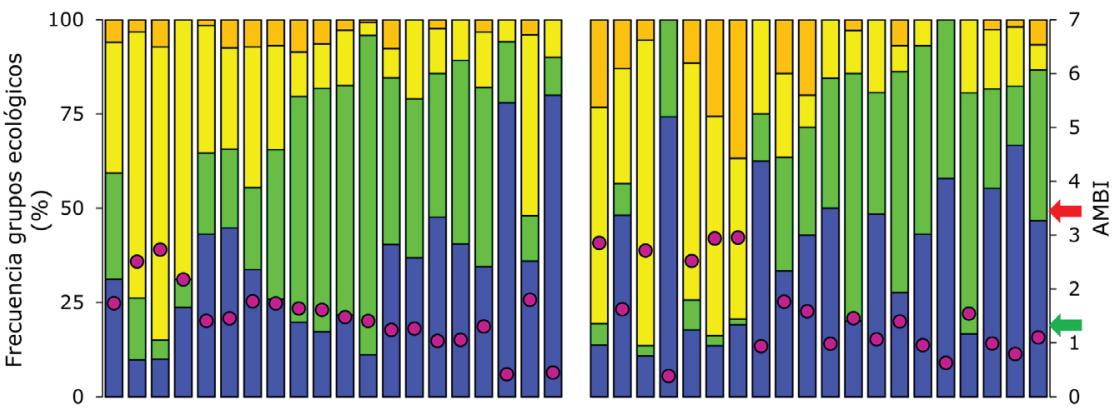

c)

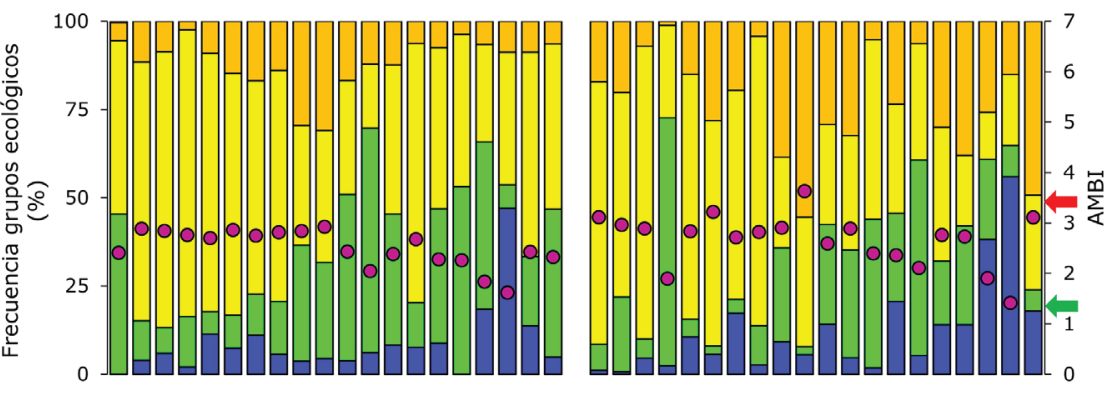

d)
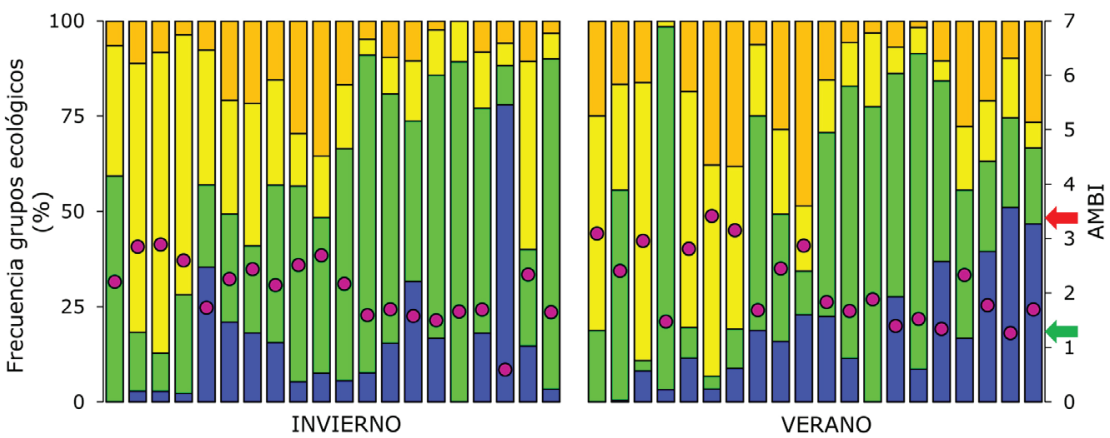

Figura 8. Estimaciones de AMBI obtenidas a partir de la abundancia de la macrofauna marina bentónica del Golfo de Arauco mediante tamices de $500 \mu \mathrm{m}(\mathrm{a}, \mathrm{c})$ y $1.000 \mu \mathrm{m}$ (b, d). Cada gráfica representa la frecuencia de los grupos ecológicos registrados (barras) y el valor del índice AMBI (círculo rojo). Los paneles de la izquierda corresponden a invierno y los de la derecha a verano. a, b) se presentan los valores estimados para $\mathrm{AMBI}-1 ; \mathrm{c}, \mathrm{d}$ ) valores obtenidos para $\mathrm{AMBI}-2$ (ver metodologías para detalles de estas estimaciones). Las flechas verdes y rojas representan el límite superior de las categorías no-contaminado y levemente contaminado de AMBI / Estimations of AMBI obtained using the abundance of marine benthic macrofauna of the Gulf of Arauco with sieve sizes of $500 \mu \mathrm{m}(\mathrm{a}, \mathrm{c})$ and $1,000 \mu \mathrm{m}(\mathrm{b}, \mathrm{d})$. Each graph shows the frequency of the ecological groups found (bars) and the value of the AMBI index (red circle). Left panels are for winter and right panels for summer. $a-b)$ estimated values for AMBI-1; c-d) values for AMBI-2 (see methods for details of these estimations). Green and red arrows represent the upper limit of no-polluted and slightly polluted categories according to $A M B I$ 
3,0 $(1.000 \mu \mathrm{m})$ (categoría, levemente contaminado; Fig. $8 \mathrm{a}, \mathrm{b})$. En el caso de AMBI-2 estos se encuentran entre 1,5 y 4,0 $(500 \mu \mathrm{m})$ y, entre 0,5 y 3,5 $(1.000 \mu \mathrm{m})$ (categorías levemente a medianamente contaminado) (Fig. 8c, d). En la Figura 9 se observa además que, para AMBI-1 y 2, la proporción de taxa pertenecientes a GE más altos se encuentran en el tamiz de $500 \mu \mathrm{m}$, y principalmente en verano (i.e., incremento del valor de AMBI y el paso desde una categoría de levemente a medianamente contaminado).
Finalmente, en el análisis PCO (Fig. 10) se representa la separación del conjunto de variables comunitarias univariadas y bio-indicadores para la disimilitud comunitaria multivariada encontrada entre las abundancias de los tamices de 500 y $1.000 \mu \mathrm{m}(75,1 \%$ de variación total explicada). De este análisis se desprende que el tamiz de $500 \mu \mathrm{m}$ es mejor representado por mayores valores de AMBI y dominancia Simpson; mientras que el de 1.000 $\mu \mathrm{m}$ es mejor representado por mayores valores de riqueza de especies/taxa, diversidad Shannon-Wiener, equidad de Pielou, índice W y M-AMBI (i.e., mejor estatus ecológico). a)

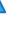

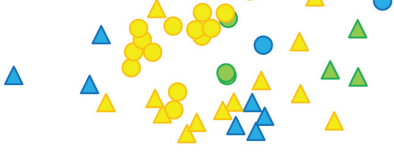

OINV I

- INV II

- INV III

$\triangle$ VER I

$\triangle$ VER II

$\triangle$ VER III

b)

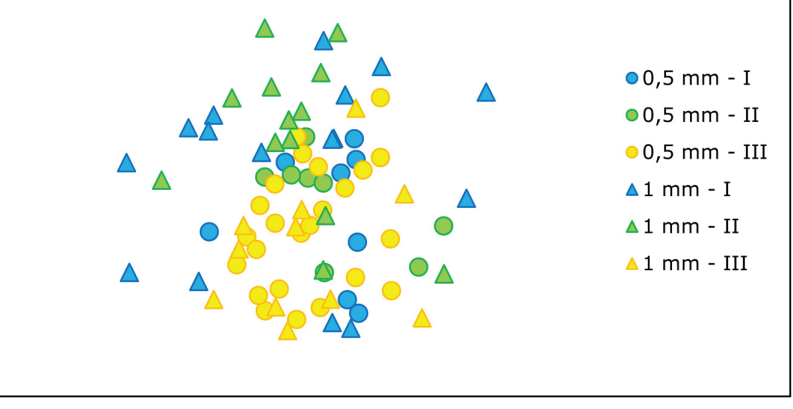

d)

- INV I

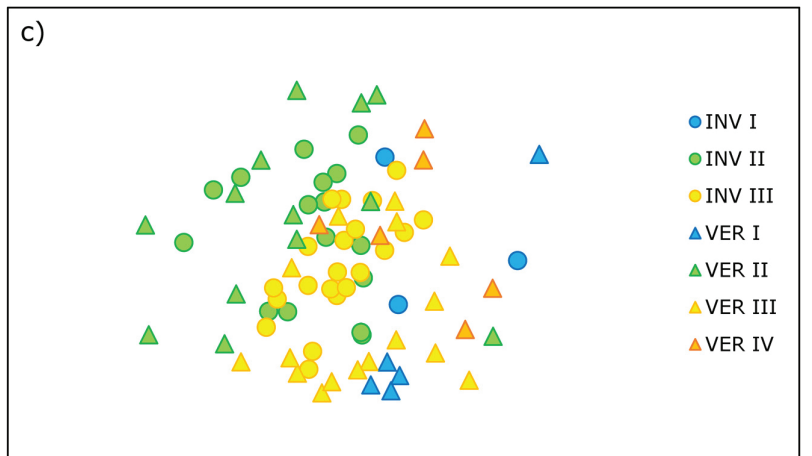

$\triangle$

- INV II

INV III

$\triangle$ VER I

$\triangle$ VER II

$\triangle$ VER III

$\triangle$ VER IV

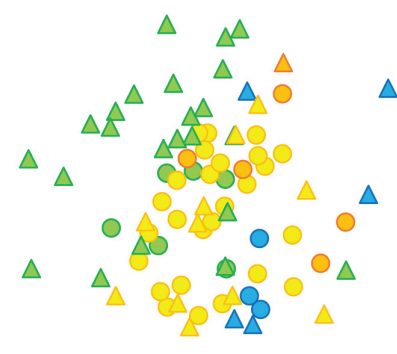

$0,5 \mathrm{~mm}$ - I

$00,5 \mathrm{~mm}$ - II

$0,5 \mathrm{~mm}$ - III

$0,5 \mathrm{~mm}$ - IV

$\Delta 1 \mathrm{~mm}-\mathrm{I}$

$\Delta 1 \mathrm{~mm}$ - II

$\triangle 1 \mathrm{~mm}$ - III

$\Delta 1 \mathrm{~mm}-\mathrm{IV}$

Figura 9. Análisis no-métrico de escalamiento multidimensional (nMDS) para las abundancias de la macrofauna marina bentónica obtenida en el Golfo de Arauco (similar nMDS de la Figura 5). Cada uno de los puntos corresponde a una de las réplicas (i.e., muestra) y el color representa al grupo ecológico dominante obtenido para esa muestra mediante AMBI-1 (a, b) y AMBI-2 (c, d). Los símbolos diferencian, además, el período de muestreo (a, c) (invierno: círculo y verano: triángulo) y, el tamiz (b, d) (500 $\mu \mathrm{m}$ : círculo y $1.000 \mu \mathrm{m}$ : triángulo). Colores de los grupos ecológicos son similares a los representados en la Figura $\mathbf{8}$ / Non-metric multidimensional scaling (nMDS) analysis for the abundance of marine benthic macrofauna obtained in the Gulf of Arauco (similar to the nMDS of Figure 5). Each point is one replica (i.e. sample); the color indicates the dominant ecological group obtained for this sample using $\mathrm{AMBI}-1(\mathrm{a}, \mathrm{b})$ and $\mathrm{AMBI}-2(\mathrm{c}, \mathrm{d})$. The symbols also indicate the sampling period $(\mathrm{a}, \mathrm{c})(\mathrm{circle}=\mathrm{winter}, \mathrm{triangle}=$ summer) and the sieve size (b, d) (500 $\mu \mathrm{m}$ : circle and 1,000 $\mu \mathrm{m}$ : triangle). Colors of the ecological groups as in Figure 8 


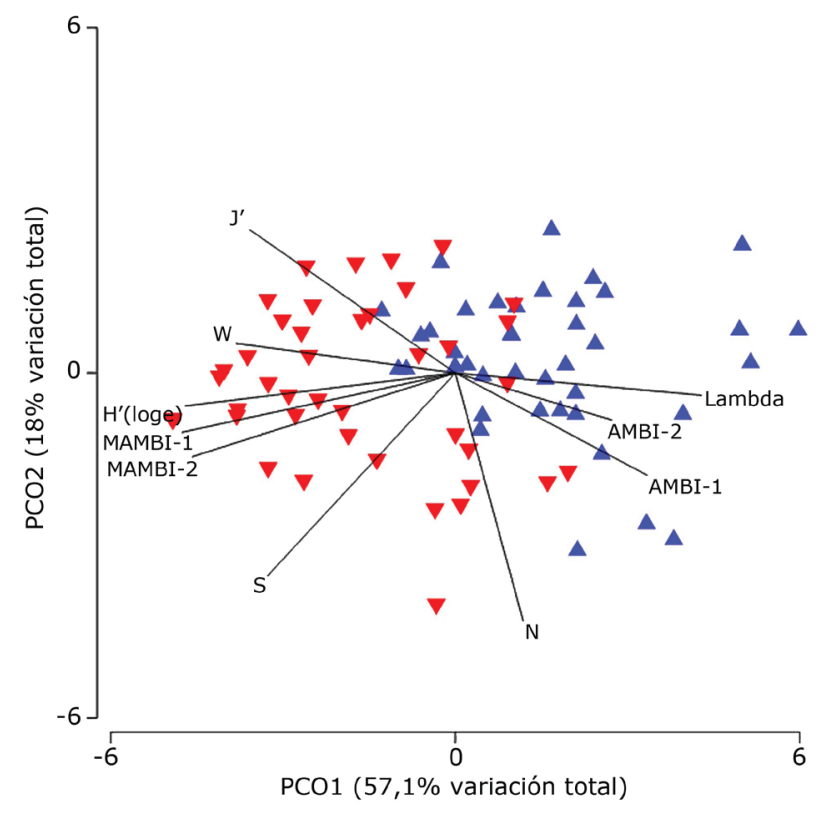

Figura 10. Análisis de ordenación de coordenadas principales (PCO) de la macrofauna marina bentónica del Golfo de Arauco obtenida mediante tamices de $500 \mu \mathrm{m}$ (símbolos azules) y $\mathbf{1 . 0 0 0} \mu \mathrm{m}$ (símbolos rojos) para el conjunto de variables señaladas en el Figura 1. Mediante vectores se señala la correlación de cada una de estas variables en relación a la distribución multivariada del conjunto de ellas. Este análisis además incorpora el índice multivariado MAMBI-1 y MAMBI-2 (ver metodologías para detalles de estas estimaciones). La gráfica da cuenta del porcentaje total de variación explicado por los ejes PCO1 y PCO2 / Principal coordinates analysis (PCO) of the benthic marine macrofauna of the Gulf of Arauco obtained with sieve sizes of $500 \mu \mathrm{m}$ (blue symbols) and 1,000 $\mu \mathrm{m}$ (red symbols) for the set of variables indicated in Figure 1 . Vectors indicate the correlation of each of these variables in relation to the multivariate distribution of all. This analysis also incorporates the multivariate indexes MAMB1-1 and MAMBI-2 (see methodology for details of these estimations). The figure also shows the percentage of the total variation explained by the PCO1 and PCO2 axes

\section{DisCUSIÓN}

\section{Patrones en la macrofauna marina bentónica}

A partir de los análisis de diversidad univariada y multivariada para la macrofauna bentónica, se infieren dos resultados principales (i) la existencia de diferencias significativas en la estructura comunitaria entre tamices, entre áreas y períodos de muestreo, (ii) cada tamiz por separado (i.e., 500 y $1.000 \mu \mathrm{m}$ ) también identifica diferencias comunitarias significativas entre áreas y periodos de muestreo. Ambos resultados permiten profundizar sobre los alcances ecológicos y ambientales de la abertura de tamiz utilizada para caracterizar este tipo de comunidades. Se observaron diferencias en los descriptores univariados de riqueza de especies/ taxa, diversidad Shannon-Wiener, equidad de Pielou y dominancia de Simpson, representando el tamiz de 500 $\mu \mathrm{m}$ una comunidad con un mayor número de taxa y mayor dominancia de algunos de ellos. Sólo la abundancia total no presentó diferencias significativas entre tamices, lo que se explica probablemente, por el amplio rango de valores registrado entre las diferentes muestras (rango desde 15 a 585 ind. $0,01 \mathrm{~m}^{-2}$ ). Sin embargo, el uso de un tamiz de $1.000 \mu \mathrm{m}$ determinó una pérdida en la abundancia total de un $67,8 \%$, valor dentro del rango (34-75\%) reportado por Rubio-Polania et al. (2016) en un estudio realizado para la macrofauna bentónica presente en el Estero de Punta Banda, Baja California, que comparó el uso de tamices de 500 y $1.000 \mu \mathrm{m}$. Desde el punto de vista multivariado, sin embargo, el cual considera las abundancias y biomasas relativas de cada uno de los taxa identificados, entregando antecedentes más detallados de la comunidad, los análisis estadísticos sí registran diferencias significativas entre tamices (i.e., presencia de una comunidad disímil entre tamices). A partir de estos resultados se concluye que en el tamiz de $500 \mu \mathrm{m}$ son retenidas en mayores abundancias taxa oportunistas y de menor tamaño. Por ejemplo, en el tamiz de $500 \mu \mathrm{m}$ los taxa más representativos en abundancia fueron Mediomastus sp., Nematoda, Carazziella sp. y Diopatra sp., mientras que en el de $1.000 \mu \mathrm{m}$ fueron Mediomastus sp., Diopatra sp., Ophiuroidea y Lumbrineris sp. A excepción de Nematoda y Ophiuroidea, todos estos taxa pertenecen al grupo de los poliquetos, siendo Mediomastus sp., perteneciente a la familia Capitellidae y Carazziella sp. a la familia Paraonidae, considerados como oportunistas. Diopatra sp., perteneciente a la familia Onuphidae y Lumbrineris sp. a la familia Lumbrineridae, son considerados como no-oportunistas, de hecho, Lumbrineris sp. es reconocido como un activo carnívoro, depredando incluso sobre 
poliquetos de menor tamaño, entre ellos Carazziella sp. (Carrasco \& Oyarzún 1988). Comparativamente, entre tamices (de $500 \mu \mathrm{m}$ a $1.000 \mu \mathrm{m}$ ), el poliqueto oportunista Mediomastus sp. baja su representatividad desde 39,2 hasta 20,4 ind. $0,01 \mathrm{~m}^{-2}$ y Carazziella sp. desde 17,4 hasta 2,1 ind. $0,01 \mathrm{~m}^{-2}$; mientras que el grupo Nematoda (taxon tolerante, también asociado a grupos oportunistas; Borja et al. 2000) baja desde 30,5 ind. $0,01 \mathrm{~m}^{-2}$ hasta 2,4 ind. $0,01 \mathrm{~m}^{-2}$, antecedentes que ponen en evidencia una sub-estimación de las abundancias relativas de especies/ taxa oportunistas y de menor tamaño al caracterizar la macrofauna marina bentónica con un tamiz de 1.000 $\mu \mathrm{m}$. De acuerdo a los análisis multivariados, diferencias en la estructura comunitaria entre áreas y períodos de muestreos también fueron identificadas por cada tamiz por separado (Tabla 4). Es decir, no obstante, las diferencias comunitarias identificadas entre tamices, ambos por separado son capaces de registrar disimilitudes para la macrofauna entre áreas y períodos de muestreo. De los resultados, se concluye que, si bien utilizando un tamiz de $1.000 \mu \mathrm{m}$ es posible identificar diferencias comunitarias espaciales y temporales de la macrofauna, en términos generales, con su uso, se sub-estiman las abundancias de taxa oportunistas y de menor tamaño, las cuales son fundamentales al utilizar bio-indicadores comunitarios de contaminación y/o perturbación ambiental.

Los ambientes marinos bentónicos están sujetos a cambios producto de forzantes de origen antropogénicos que afectan a las poblaciones y comunidades, con una amplia variedad de respuestas; por ejemplo, producto del enriquecimiento orgánico, el cual favorece preferentemente la presencia de ciertas especies oportunistas (Pearson \& Rosenberg 1978, Warwick 1986). Estas especies corresponden a organismos colonizadores que dominan las etapas tempranas de una sucesión ecológica posterior a una perturbación y, en muchos casos suelen ser de sólo unos pocos taxa (Dauer \& Simon 1976, Zajac \& Whitlatch 1982). Entre ellos se encuentran algunos poliquetos, quienes se ven favorecidos por su capacidad de proliferar rápidamente luego de un incremento en la concentración de materia orgánica en los sedimentos; destacando, por ejemplo, especies pertenecientes a las familias Capitellidae, Spionidae y Cirratulidae (Pearson \& Rosenberg 1978, Glémarec \& Hily 1981), ampliamente reconocidas como bio-indicadoras de contaminación y que presentan pequeños tamaños corporales (Grassle \& Grassle 1974, Zajac \& Whitlatch 1982, Whitlatch \& Zajac 1985, Cognetti \& Maltagliati 2000). Desde un punto de vista ecológico, se ha sugerido el uso de estas familias de poliquetos oportunistas como bio-indicadores en monitoreos ambientales, producto de su valor particular como indicadores de contaminación (Pocklington \& Wells 1992, Dean \& Blake 2009). Por ejemplo, Rivero et al. (2005), en un estudio de gradientes espaciales de la macrofauna en el Puerto de Mar del Plata, señalan a Capitella spp. como un bio-indicador de condiciones ambientales perturbadas (i.e., con alta concentración de materia orgánica, de hidrocarburos y baja hidrodinámica), asociando sus altas abundancias a una baja diversidad. Plantean que, áreas medianamente perturbadas, mantendrían la presencia de C. capitata y Capitella sp., además de Polydora sp., Tharyx sp. y Mediomastus sp.; mientras que áreas menos perturbadas, serían caracterizadas por la presencia de Mediomastus sp., especies de la familia Maldanidae y por Aglaophamus uruguayi. De estos resultados, se puede inferir que, lo planteado por Pocklington \& Wells (1992), sobre que sólo la presencia de algunos grupos taxonómicos de poliquetos como Capitellidae y Spionidae, serían indicadores de contaminación en un área particular, pueden inducir a subestimar y sobre simplificar los amplios márgenes de historia de vida de algunos grupos de especies estrechamente relacionados (Dean \& Blake 2009). Por lo tanto, incorporar análisis comunitarios y no sólo de algunos taxa o grupos específicos, y utilizando gradientes espaciales respecto a una fuente de contaminación, debiera ser considerado metodológicamente.

Por otro lado, la variabilidad temporal en las abundancias de estos organismos oportunistas también debe ser considerada como un factor relevante en los análisis. Por ejemplo, Elías et al. (2005) describen al espiónido, Prionospio sp., especie indicadora de enriquecimiento orgánico, con una alta abundancia durante la temporada de verano cerca de la ciudad de Mar del Plata en Argentina. Los autores señalan además que, la disminución invernal de turistas en la zona reduce considerablemente el aporte de materia orgánica a estas aguas costeras, lo que, complementado con la presencia de tormentas, ayudarían a la disminución de su concentración, determinando finalmente un descenso en las abundancias de la especie. Así, en el período de baja carga orgánica, en dicha zona, las especies de poliquetos dominantes correspondieron a taxa de la familia Maldanidae. Al contrario, la abundancia del cirratúlido Caulleriella sp., que habría estado casi completamente ausente en invierno, presentaría altas abundancias durante el verano (Elías et al. 2004, 2005), asignándole también un rol como bio-indicador de enriquecimiento orgánico. Ambos estudios (Elías et al. 2004, 2005) ejemplifican la capacidad de los poliquetos para responder rápidamente (i.e., meses) a los cambios en su entorno, lo que sugiere que no sólo las especies dominantes y tolerantes actuarían como bio-indicadores de las condiciones ambientales en el bentos y que un enfoque comunitario temporal también sería más adecuado. Por otro lado, fuertes cambios poblacionales y comunitarios temporales de la macrofauna bentónica marina de Chile centro-sur también pueden ser producidos 
por perturbaciones naturales como terremotos-tsunamis (Cárcamo et al. 2017) y gradientes en los niveles de oxígeno disuelto en la columna de agua (Veas et al. 2012), cambios descritos para la Bahía de Concepción y la Plataforma del Itata, respectivamente. Así, el cambio en la estructura comunitaria entre invierno y verano detectado en este estudio realizado en el Golfo de Arauco ratifica la existencia de una variabilidad temporal intrínseca en este grupo de organismos y, la necesidad de su cuantificación espacial en gradientes a distintas escalas temporales al momento de ser utilizados como un bio-indicador. El uso de un tamiz de $500 \mu \mathrm{m}$ por lo demás, garantiza una mejor cuantificación de las abundancias de las especies/taxa oportunistas, características de ambientes perturbados, principalmente por enriquecimiento orgánico.

\section{MACROFAUNA MARINA BENTÓNICA COMO BIO- INDICADOR COMUNITARIO}

En relación al estatus ecológico obtenido a partir de la macrofauna bentónica, el índice AMBI (que consideró una estrategia de mejor y peor asignación de GE), entregó diferencias significativas, entre tamices, entre áreas y períodos de muestreo (Figs. 1, 2 y 8). El índice de W, también entregó diferencias significativas entre tamices, áreas y períodos de muestreo. En general ambos índices asignan un más bajo estatus ecológico a las muestras obtenidas en el tamiz de $500 \mu \mathrm{m}$. Estos resultados permiten inferir que la elección de un tamiz de $1.000 \mu \mathrm{m}$ para caracterizar la macrofauna, reduciría su eficiencia como un bio-indicador comunitario, producto de la cuantificación de una comunidad disímil (i.e., sub-estimación de las abundancias de especies oportunistas y de menor tamaño) a la realmente existente en el área bajo estudio. Por ejemplo, en el caso de $\mathrm{W}$, los valores estimados disminuyen desde un rango promedio de 0,29-0,31 (1.000 $\mu \mathrm{m})$ a 0,24-0,25 $(500 \mu \mathrm{m}) \mathrm{y}$, en el caso de AMBI-2, los rangos aumentan desde 0,5-3,5 $(1.000 \mu \mathrm{m})$ a 1,5-4,0 $(500 \mu \mathrm{m})$ (Figs. 8 y $9)$, esto como resultado del incremento en la proporción de las abundancias de especies/taxa pertenecientes a GE más altos en el tamiz de $500 \mu \mathrm{m}$, y principalmente en el muestreo de verano, período asociado probablemente a un mayor reclutamiento de juveniles (i.e., descenso de los tamaños corporales y aumento de especies oportunistas). En específico para AMBI, estos resultados determinan que algunas muestras de la macrofauna, dependiendo del tamiz utilizado, cambian desde una categoría de "levemente contaminado en $1.000 \mu \mathrm{m}$ " a una "medianamente contaminado en $500 \mu \mathrm{m}$ ". En este contexto de estatus ecológico, y enmarcado dentro del principio precautorio, se sugiere, además, utilizar el criterio de AMBI-2 (como fue ejemplificado en este estudio) al momento de asignar taxones a un GE en el caso de que inicialmente no se encuentren en el listado de AMBI. Este criterio permitiría determinar los valores más altos del rango esperable del bio-indicador, estimado a partir de la comunidad de la macrofauna bentónica identificada en un área particular de estudio mediante un tamiz de $500 \mu \mathrm{m}$. Si bien, para esta zona de estudio, los valores estimados para AMBI no se encuentran en el rango alto del indicador (áreas levemente o moderadamente contaminadas, i.e., cercanas al límite $3,3)$, es esperable que mayores diferencias entre tamices para ambos índices (i.e., incremento de AMBI y reducción de W) sean observadas en ambientes con una alta carga de materia orgánica, con sedimentos altamente reducidos.

En Chile, Pino et al. (2015) evaluaron el uso de dos aberturas de trama de tamices para la caracterización de la macrofauna bentónica al suroeste del Seno de Reloncaví. Este estudio, basado en muestras cercanas y lejanas a dos centros de cultivo de mitílidos, reporta que, de manera similar a lo registrado por el nuestro, el uso de un tamiz de $500 \mu \mathrm{m}$ aumenta la abundancia de especies/taxa oportunistas, en comparación a uno de $1.000 \mu \mathrm{m}$. Sus resultados, sin embargo, no detectaron diferencias significativas en el índice AMBI entre tamices Sus resultados, sin embargo, no detectaron diferencias significativas en el índice AMBI entre tamices (rango de 1,07 a 3,97 en $500 \mu \mathrm{m}$ y de 1,17 a 4,07 $1.000 \mu \mathrm{m}$ ), señalando además que la información generada por el tamiz de $500 \mu \mathrm{m}$ no mejoró la detección de diferencias comunitarias univariadas entre las áreas de referencia y las potencialmente impactadas por los cultivos de mitílidos. Por último, estos autores señalan que el uso de un tamiz de $500 \mu \mathrm{m}$ sí ayudaría a incrementar el número de taxa, y, la densidad y biomasa de especies raras, razón por la cual, bajo un enfoque precautorio, sugieren utilizar esta abertura de tamiz en estudios de CPS para la acuicultura. A diferencia de Pino et al. (2015), basado sólo en descriptores univariados, el presente estudio, que incorpora además análisis estadísticos multivariados, ampliamente sugeridos para realizar estudios comunitarios (Clarke \& Warwick 2001, Clarke \& Gorley 2006, Anderson et al. 2008) y bio-indicadores, sí detectó diferencias significativas para la macrofauna entre tamices (Tablas 4 y 5), sugiriendo un estatus ecológico más bajo en muestras obtenidas en el tamiz de $500 \mu \mathrm{m}$. Con el objetivo de interpretar el conjunto de variables obtenidas con una aproximación multivariada, se integró toda la información en un análisis PCO (Fig. 10). Este análisis permite visualizar la disimilitud de la macrofauna presente entre tamices, incorporando además el conjunto de variables ecológicas univariadas y los bio-indicadores comunitarios. Así, a partir del análisis PCO, la macrofauna presente en el tamiz de $500 \mu \mathrm{m}$ fue caracterizada por presentar valores más altos de AMBI y dominancia de Simpson; mientras que la presente en 
el tamiz de $1.000 \mu \mathrm{m}$ estuvo caracterizada por mayores valores de riqueza de especies/taxa, de diversidad Shannon-Wiener, y de los bio-indicadores W y M-AMBI, sugiriendo en conjunto un más alto estatus ecológico de la comunidad.

Si bien, utilizar un tamiz de $1.000 \mu \mathrm{m}$ (como lo señala la metodología chilena ambiental actual en la Res. Ex. N³612), agiliza el análisis de muestras y disminuye los esfuerzos de identificación y clasificación taxonómica (Kingston \& Riddle 1989, Lampadariou et al. 2005) obteniendo resultados descriptivos a un menor costo (Ferraro et al. 1989, Lampadariou et al. 2005, Couto et al. 2010), los resultados obtenidos sugieren que esta metodología debiera utilizarse en estudios que no tienen como objetivo evaluar impactos de origen antropogénico, y en particular por enriquecimiento orgánico. En conclusión, si el objetivo del estudio es evaluar impactos en el ambiente o el estatus ecológico a partir de la macrofauna bentónica, se debiera utilizar un tamiz de $500 \mu \mathrm{m}$, con lo cual se minimizaría la sub-estimación de especies/taxa bio-indicadoras y oportunistas, generando una base de datos taxonómica de mejor resolución. Se recomienda el uso de un tamiz de $500 \mu \mathrm{m}$ especialmente en Líneas de Base o CPS para la Acuicultura, Monitoreos y/o Programas de Vigilancia Ambiental asociados al Sistema de Evaluación de Impacto Ambiental vigente en Chile. Esta información, debería estar complementada con una batería de herramientas estadísticas multivariadas, como las aquí presentadas, que sean capaces de incorporar toda la información biológica y ambiental recopilada. Estas consideraciones metodológicas permitirían una mejora cuantitativa en la utilización de bio-indicadores comunitarios, necesarios para la evaluación adecuada de impactos antropogénicos en el ambiente marino, y requeridos por los organismos públicos, en la normativa chilena ambiental.

\section{Agradecimientos}

Esta investigación fue financiada por: (i) Colbún S.A. en el marco del denominado "Plan de Vigilancia Ambiental del Complejo Santa María de Coronel", (ii) Programa de Estudios Ecosistémicos del Golfo de Arauco (PREGA) de la Facultad de Ciencias Naturales y Oceanográficas de la Universidad de Concepción financiado por Celulosa Arauco y Constitución S.A., y (iii) Centro Interdisciplinario para la Investigación Acuícola (INCAR) financiado por FONDAP/ ANID ( $\left.{ }^{\circ} 15110027\right)$. Los autores desean entregar sinceros agradecimientos al Editor Asociado MFLD y a los cuatro revisores anónimos, cuyos comentarios permitieron fortalecer esta publicación.

\section{LITERATURA CITADA}

Anderson MJ. 2001. A new method for non-parametric multivariate analysis of variance. Austral Ecology 26: 32-46.

Anderson M, K Ellingsen \& B McArdle. 2006. Multivariate dispersion as a measure of beta diversity. Ecology Letters 9: 683-693.

Anderson MJ, RN Gorley \& KR Clarke. 2008. PERMANOVA+ for PRIMER: Guide to software and statistical methods, $214 \mathrm{pp}$. PRIMER-E, Plymouth.

Anderson MJ, TO Crist, JM Chase, M Vellend, BD Inouye, AL Freestone, NJ Sanders, HV Cornell, LS Comita, KF Davies, SP Harrison, NJB Kraft, JC Stegen \& NG Swenson. 2011. Navigating the multiple meanings of $\beta$ diversity: a roadmap for the practicing ecologist. Ecology Letters 14: 19-28.

Andrew NL \& BD Mapstone. 1987. Sampling and the description of spatial pattern in marine ecology. Oceanography and Marine Biology, An Annual Review 25: 39-90.

Barba B, A Larranaga, A Otermin, A Basaguren \& J Pozo. 2010. The effect of sieve mesh size on the description of macroinvertebrate communities. Limnetica 29: 211-220.

Bachelet G. 1990. The choice of a sieving mesh size in the quantitative assessment of marine macrobenthos: a necessary compromise between aims and constraints. Marine Environmental Research 30: 21-35.

Beauchard O, H Veríssimo, AM Queirós \& PMJ Herman. 2017. The use of multiple biological traits in marine community ecology and its potential in ecological indicator development. Ecological Indicators 76: 81-96.

Bilyard GR. 1987. The value of benthic infauna in marine pollution monitoring studies. Marine Pollution Bulletin 18: 581-585.

Bishop JDD \& JP Hartley. 1986. A comparison of the fauna retained on $0.5 \mathrm{~mm}$ and $1 \mathrm{~mm}$ meshes from benthic samples taken in the Beatrice Oilfield, Moray Firth, Scotland. Proceedings of the Royal Society of Edinburgh 91B: 247-262.

Borja A \& I Muxica. 2005. Guidelines for the use of AMBI (AZTI's Marine Biotic Index) in the assessment of the benthic ecological quality. Marine Pollution Bulletin 50: 787-789.

Borja A, J Franco \& V Pérez. 2000. A marine biotic index to establish the ecological quality of soft-bottom benthos within European estuarine and coastal environments. Marine Pollution Bulletin 40: 1100-1114.

Borja A, J Mader \& I Muxika. 2012. Instructions for the use of the AMBI index software (Version 5.0). Revista de Investigación Marina, AZTI-Tecnalia 19(3): 71-82.

Cárcamo PJ, E Hernández-Miranda, R Veas \& R Quiñones. 2017. Macrofaunal community structure in Bahía Concepción (Chile) before and after the $8.8 \mathrm{Mw}$ Maule mega-earthquake and tsunami. Marine Environmental Research 130: 233-247.

Carey JM \& MJ Keough. 2002. Compositing and subsampling to reduce costs and improve power in benthic infaunal monitoring programs. Estuaries 25(5): 1053-1061. 
Carrasco FD \& VA Gallardo. 1989. La contaminación marina y el valor de la macroinfauna en su evaluación y vigilancia: casos de estudio en el litoral de Concepción. Chile. Biología Pesquera 18: 15-27.

Clarke KR. 1990. Comparisons of dominance curves. Journal of Experimental Marine Biology and Ecology 138: 143157.

Clarke KR \& RN Gorley. 2006. PRIMER v6: User manual/ tutorial, 190 pp. PRIMER-E, Plymouth.

Clarke KR \& RM Warwick. 2001. Change in marine communities: an approach to statistical analysis and interpretation, 172 pp. PRIMER-E, Plymouth.

Cognetti G \& F Maltagliati. 2000. Biodiversity and adaptive mechanisms in brackish-water fauna. Marine Pollution Bulletin 40: 7-14.

Couto T, J Patricio, J Neto, F Ceia, J Franco \& J Marques. 2010. The influence of mesh size in environmental quality assessment of estuarine macrobenthic communities. Ecological Indicators 10: 1162-1173.

Dauer DM \& JL Simon. 1976. Habitat expansion among polychaetous annelids repopulating a defaunated marine habitat. Marine Biology 37: 169-177.

Dauvin JC. 2007. Paradox of estuarine quality: Benthic indicators and indices. Consensus or debate for the future. Marine Pollution Bulletin 55: 271-281.

Dauvin JC \& T Ruellet. 2007. Polychaete/amphipod ratio revisited. Marine Pollution Bulletin 55: 215-224.

Dauvin JC. G Bellan \& D Bellan-Santini. 2010. Benthic indicators: From subjectivity to objectivity - Where is the line? Marine Pollution Bulletin 60: 947-953.

Dean HK \& JA Blake. 2009. Monticellina (Polychaeta: Cirratulidae) from the Pacific coast of Costa Rica with description of six new species. Zoosymposia 2: 105-126.

de Souza GBG \& F Barros. 2017. Cost/benefit and the effect of sample preservation procedures on quantitative patterns in benthic ecology. Helgoland Marine Research 71:21 $<$ DOI 10.1186/s10152-017-0501-3>

Edgar GJ. 1990. The use of size structure of benthic macrofaunal communities to estimate faunal biomass and secondary production. Journal of Experimental Marine Biology and Ecology 137: 195-214.

Eleftheriou A \& NA Holme. 1984. Macrofauna techniques. In: Holme NA \& AD McIntyre (eds). Methods for the study of marine benthos, pp. 140-216. Blackwell. Oxford.

Elías R, EA Vallarino, M Scagliola \& FI Isla. 2004. Macrobenthic distribution patterns at a sewage disposal site in the inner shelf off Mar del Plata (SW Atlantic). Journal of Coastal Research 20: 1176-1182.

Elías R, JR Palacios, MS Rivero \& EA Vallarino. 2005. Short-term responses to sewage discharge and storms of subtidal sand-bottom macrozoobenthic assemblages off Mar del Plata City, Argentina (SW Atlantic). Journal of Sea Research 53: 231-242.

Ferraro SP, FA Cole, WA DeBen \& RC Swartz. 1989. Powercost efficiency of eight macrobenthic sampling schemes in Puget Sound, Washington, USA. Canadian Journal of Fisheries and Aquatic Sciences 46: 2157-2165.

Glémarec M \& C Hily. 1981. Perturbations apportées à la macrofaune benthique de la baie de Concarneau par les effluents urbains et portuaires. Acta Oecologica 2: 139-150.
Grall J \& M Glémarec. 1997. Using biotic indices to estimate macrobenthic community perturbations in the Bay of Brest. Estuarine, Coastal and Shelf Science 44: 43-53.

Grassle JF \& JP Grassle. 1974. Opportunistic life-histories and genetic systems in marine benthic polychaetes. Journal of Marine Research 32: 253-284.

Gray JS, MR Carr, KR Clarke, RH Green, TH Pearson, R Rosenberg \& RM Warwick. 1988. Analysis of community attributes of the benthic macrofauna of Frierfjord/ Langesundfjord and in a mesocosm experiment. Marine Ecology Progress Series 46: 151-165.

Green RH. 1979. Sampling design and statistical methods for environmental biologists, $272 \mathrm{pp}$. Wiley-Interscience, Chichester.

Harris PT. 2012. Anthropogenic threats to benthic habitats. In: Harris PT \& EK Baker (eds). Seafloor geomorphology as benthic habitat, pp. 39-60. Elsevier, Amsterdam.

Hily C, H Le Bris \& M Glémarec. 1986. Impacts biologiques des émissaires urbains sur les écosystémes benthiques. Oceanis 12: 419-426.

Hudson PL \& JV Adams. 1998. Sieve efficiency in benthic sampling as related to chironomid head capsule width. Journal of the Kansas Entomological Society 71: 456-468.

James RJ, MP Lincoln-Smith, PG Fairweather. 1995. Sieve mesh-size and taxonomic resolution needed to describe natural spatial variation of marine macrofauna. Marine Ecology Progress Series 118: 187-198.

Kennedy CJ \& SM Cheong. 2013. Lost ecosystem services as a measure of oil spill damages: a conceptual analysis of the importance of baselines. Journal of Environmental Management 128: 43-51.

Kennelly SJ \& AJ Underwood. 1984. Underwater microscope sampling of a sublittoral kelp community. Journal of Experimental Marine Biology and Ecology 76: 67-78.

Kingsford MJ, CN Battershill \& K Walls. 1998. Introduction to ecological assessments. In: Kingsford M \& C Battershill (eds). Studying temperate marine environments - a handbook for ecologists, pp. 17-28. Canterbury University Press, Christchurch.

Kingston PF \& MJ Riddle. 1989. Cost effectiveness of benthic faunal monitoring. Marine Pollution Bulletin 20: 490-496.

Lampadariou N, I Karakassis \& TH Pearson. 2005. Cost/ benefit analysis of a benthic monitoring programme of organic benthic enrichment using different sampling and analysis methods. Marine Pollution Bulletin 50: 1606-1618.

Levin LA \& JD Gage. 1998. Relationships between oxygen organic matter and the diversity of bathyal macrofauna. Deep Sea Research II 45: 129-163.

Levin LA, DF Boesch, A Covish, C Dahm, C Erseus, KC Ewel, RT Kneib, A Moldenke, MA Palmer, P Snelgrove, D Strayer \& JM Weslawski. 2001. The function of marine critical transition zones and the importance of sediment biodiversity. Ecosystems 4: 430-451.

Magurran AE. 2004. Measuring biological diversity, 215 pp. Blackwell Publishing, Oxford.

Marques JC, MJ Costa \& V de Jonge. 2013. Introducing "Assessing ecological quality in estuarine and coastal systems - Management perspective". Ocean \& Coastal Management 72: 1-2. 
Morales N. 2011. ¿Qué es un bioindicador? Aprendiendo a partir del ciclo de indagación guiada con macroinvertebrados bentónicos. Propuesta Metodológica. Trabajo de Investigación para optar al título de Magister en Enseñanza de las Ciencias Exactas y Naturales, Universidad Nacional de Colombia, Facultad de Ciencias, Leticia, 48 pp. $<$ http://bdigital.unal. edu.co/10195/1/naferedivarmoralessalinas.2011.pdf $>$

Morin A, J Stephenson, J Strike \& A Solimini. 2004. Sieve retention probabilities of stream benthic invertebrates. Journal of the North American Benthological Society 23: 383-391.

Muxika I, A Borja \& J Bald. 2007. Using historical data, expert judgment and multivariate analysis in assessing reference conditions and benthic ecological status, according to the European Water Framework Directive. Marine Pollution Bulletin 55: 16-29.

Paul JF, KJ Scott, DE Campbell, JH Gentile, CS Strobel, RM Valente, SB Weisberg, AF Holland \& JA Ranasinghe. 2001. Developing and applying a benthic index of estuarine condition for the Virginian Biogeographic Province. Ecological Indicators 1: 83-99.

Pearson TH \& R Rosenberg. 1978. Macrobenthic succession in relation to organic enrichment and pollution of the marine environment. Oceanography and Marine Biology: An Annual Review 16: 229-311.

Pino L, SL Marín \& R Nuñez. 2015. Indicadores bióticos y fracción de tamaños en la definición de la macrofauna. Latin American Journal of Aquatic Research 43(2): 329-336.

Pocklington P \& PG Wells. 1992. Polychaetes key taxa for marine environmental quality monitoring. Marine Pollution Bulletin 24: 593-598.

Ramsay PM, SD Rundle, MJ Attrill, MG Uttley, PR Williams, PS Elsmere \& A Abada. 1997. A rapid method for estimating biomass size spectra of benthic metazoan communities. Canadian Journal of Fisheries and Aquatic Sciences 54: 1716-1724.

Reish DJ. 1959. A discussion of the importance of the screen size in washing quantitative marine bottom samples. Ecology 40: 307-309.

Rivero MS, R Elías \& EA Vallarino. 2005. First survey of macrofauna in the Mar del Plata Harbor (Argentina), and the use of polychaetes as pollution indicators. Revista de Biología Marina y Oceanografía 40: 101-108.

Rubio-Polania JC, E Solana-Arellano, V Díaz-Castañeda \& O Flores-Uzeta. 2016. Efecto del tamaño de malla en los valores de densidad y biomasa de la comunidad bentónica estuarine del Estero de Punta Banda, Baja California. Revista de Biología Marina y Oceanografía 51: 599-608.
Scheiner SM \& MR Willig. 2008. A general theory of ecology. Theoretical Ecology 1: 21-28.

Schlacher TA \& TH Wooldridge. 1996. How accurately can retention of benthic macrofauna by a particular mesh size be predicted from body size of organisms? Hydrobiologia 323: 149-154.

Solana-Arellano E, V Díaz-Castañeda, O Flores-Uzeta, H Echavarria-Heras \& JC Rubio-Polaína. 2014. Assessment of secondary production and efficiency of different mesh sizes to study benthic communities associated to a Zostera marina Meadow. Annual Research \& Review in Biology 4: 2603-2616.

Souza GBG \& F Barros. 2015. Analysis of sampling methods of estuarine benthic macrofaunal assemblages: sampling gear, mesh size, and taxonomic resolution. Hydrobiologia 743: 157-174.

Tanaka MO \& FPP Leite. 1998. The effect of sieve mesh size on the abundance and composition of macrophyteassociated macrofaunal assemblages. Hydrobiologia 389: 21-28.

Teixeira H, SB Weisberg, A Borja, JA Ranasinghe, DB Cadien, RG Velarde, LL Lovell, D Pasko, CA Phillips, DE Montagne, KJ Ritter, F Salas \& JC Marques. 2012. Calibration and validation of the AZTI's Marine Biotic Index (AMBI) for Southern California marine bays. Ecological Indicators 12: 84-95.

Veas R, E Hernández-Miranda, RA Quiñones \& FD Carrasco. 2012. Spatio-temporal biodiversity of soft bottom macrofaunal assemblages in shallow coastal waters exposed to episodic hypoxic events. Marine Environmental Research 78: 1-14.

Warwick RM. 1986. A new method for detecting pollution effects on marine macrobenthic communities. Marine Biology 92: 557-562.

Warwick RM \& KR Clarke. 1994. Relearning the ABC: taxonomic changes and abundance/biomass relationships in disturbed benthic communities. Marine Biology 118: 739-744.

Whitlatch RB \& RM Zajac. 1985. Biotic interactions among estuarine infaunal opportunistic species. Marine Ecology Progress Series 21: 299-311.

Word JQ. 1979. The infaunal trophic index. In: Bascom W (ed). Southern California Coastal Water Research Project, Annual Report 1978, pp. 19-39. Southern California Coastal Water Research Project, El Segundo.

Zajac RN \& RB Whitlatch. 1982. Response of estuarine infauna to disturbance. II. Spatial and temporal variation of succession. Marine Ecology Progress Series 10: 15-27. 